Décadrages Décadrages

cınéma, à travers champs Cinéma, à travers champs

8-9| 2006

Le monde de Star Wars

\title{
Introduction : des films, un monde. Lectures de
}

Star Wars

Alain Boillat

\section{(2) OpenEdition}

Journals

Édition électronique

URL : http://journals.openedition.org/decadrages/273

DOI : $10.4000 /$ decadrages. 273

ISSN : 2297-5977

Éditeur

Association Décadrages

Édition imprimée

Date de publication : 10 octobre 2006

Pagination : 9-41

ISBN : 978-2-9700582-4-3

ISSN : 2235-7823

Référence électronique

Alain Boillat, «Introduction : des films, un monde. Lectures de Star Wars », Décadrages [En ligne],

8-9 | 2006, mis en ligne le 04 février 2014, consulté le 01 mai 2019. URL : http://

journals.openedition.org/decadrages/273 ; DOI : 10.4000/decadrages.273

(B) Décadrages 


\section{INTRODUCTION}

Des films, un monde. Lectures de Star Wars

\section{par Alain Boillat}

Alors que le champ francophone des études sur le cinéma se montre généralement quelque peu hermétique aux approches de type cultural studies qui ne s'embarrassent pas de la légitimation «artistique» des films, une tentative de prendre en compte les trilogies de La Guerre des étoiles en tant qu'objet d'étude académique a été récemment entreprise par Laurent Jullier dans l'ouvrage Star Wars. Anatomie d'une saga 1 . Bien que l'auteur témoigne parfois d'une position ambiguë envers l'adéquation de son appareil théorique à ce type de productions cinématographiques "grand public" 2 , l'intérêt de son étude réside notamment dans une confrontation directe avec les films - tous sauf l'épisode III, qui n'était pas encore sorti - à travers des analyses détaillées. Jullier n'en reste donc pas au "phénomène Star Wars» dont il tente néanmoins de prendre la mesure - les six films de la série ont suscité un fanship d'une ampleur sans précédent dans l'histoire du cinéma ${ }^{3}$-, mais s'interroge également sur des problématiques esthétiques et narratologiques. C'est dans une même volonté de considérer les épisodes et le monde de Star Wars de l'extérieur, de les construire en tant qu'«objet» - alors que la grande majorité des discours portant sur eux émane de l'intérieur (c'est-à-dire des instances de production ou de fans immergés dans l'univers) - que les articles de ce dossier se proposent, en examinant certains points laissés dans l'ombre par Jullier (n'ayant pas attendu la sortie du dernier épisode, il ne peut saisir totalement les effets de clôture et de rappel provoqués par les prequels), d'étudier le fonctionnement de cette fiction dans les rapports qu'elle instaure avec la réalité des discours et des faits culturels, notamment en s'interrogeant sur des phénomènes d'intermédialité. Dans cette perspective, on s'attachera également à des manifestations périphériques aux films comme les jeux vidéo et les produits dérivés, qui se sont approprié à leur manière l'univers élaboré au cours des deux trilogies. Par-delà la diversité des approches et des sujets, nous serons constamment amenés à discuter le caractère ambivalent des liens qui
1 Laurent Jullier, Star Wars. Anatomie d'une saga, Armand Colin, Paris, 2005.

2 Soucieux de s'adresser à un public large tout en témoignant d'une rigueur théorique (si ce n'est dans des errances bien peu productives relatives à l'arrière-plan philosophico-mystique des films), Jullier semble en effet s'excuser d'employer un vocabulaire spécialisé, alors que ce dernier n'est pas nécessairement requis dans le contexte: ainsi, il évoque des films "que l'on appelle en jargon universitaire des récits érotématiques" (p. 19) sans référence aucune à un emploi précédent de cette appellation; il précise à propos du caractère linéaire des histoires de Star Wars: "en jargon encore, disons que c'est une conception très syntagmatique du récit " (p. 21); enfin, il utilise les notions d'" anaphore" et d'"isotopie" en faisant remarquer qu'il s'agit d'uétiquettes bien savantes pour des choses bien évidentes" (p. 25).

3 Sur ce phénomène des communautés de fans, et plus précisément à propos de leur manifestation via Internet, on lira Will Brooker, "Internet Fandom and the Continuing Narratives of Star Wars, Blade Runner and Alien", in Annette Kuhn (éd.), Alien Zone II. The Spaces of Science Fiction Cinema, Verso, Londres, 1999. En ce qui concerne Star Wars, Brooker met l'accent sur l'importance des spéculations narratives qui animent les fans captivés par l'anticipation du prequel (id., p. 57). Notons que les commentaires des fans révèlent bien souvent une connaissance extrêmement pointue qui ne s'accompagne pas toujours, comme on pourrait l'imaginer a priori, d'une absence d'acuité analytique. 
4 Laurent Jullier, op. cit., pp. 153-162. Jullier n'indiquant toutefois aucune référence, son travail n'est pas vraiment utilisable.

5 Voir le chapitre "La production industrielle des biens culturelles", in La Dialectique de la raison, Gallimard, Paris, 1974 (1944).

6 Robin Wood, Hollywood from Vietnam to Reagan, Columbia University Press, New York, 1986, pp. 162-174.

7 Notamment en raison de l'appropriation par Reagan de l'expression "Star Wars" pour qualifier son projet de défense. On lira sur ce point la partie liminaire de l'article de Will Brooker, "New Hope: The Postmodern Project of Star Wars" (1996), repris in Sean Redmond (éd.), Liquid Metal. The Science Fiction Film Reader, Wallflower Press, Londres, 2004. En France, un article caractéristique de cette réception critique est celui de Serge Le Péron, "L’Amérique sans peur et sans reproche (Star Wars, G. Lucas)", in Cahiers du cinéma, n 283, décembre 1977.

8 Empire of Dreams: The Story of the "Star Wars" Trilogy (Edith Becker et Kevin Burns, 2004). Bien que livré en supplément du coffret DVD édité en 2004, ce documentaire télévisuel est plus qu'une servile hagiographie du cinéaste. II participe certes au mythe du "génie" de l'auteur, mais fournit nombre d'informations importantes sur la genèse des films. s'établissent dans Star Wars entre culture de masse et culture légitimée. Cette mixité transparaît symptomatiquement au niveau des modèles musicaux dont est imprégné le travail du compositeur John Williams sur les six films, ainsi que l'examine Laurent Guido dans son article.

Jullier consacrant une section de son livre à un bilan historiographique de la réception critique des films en France ${ }^{4}$, je me bornerai ici à évoquer quelques caractéristiques des deux trilogies qui en rendent la lecture singulière, et dont l'analyse m'apparaît féconde pour aborder sur le plan des représentations et de la narration nombre de productions hollywoodiennes contemporaines. On verra que l'intérêt a tôt fait de se déplacer des films de George Lucas proprement dits vers le monde qu'ils proposent, ce dernier étant diffusé à grande échelle par le truchement de multiples produits de consommation : romans, bandes dessinées, jeux de société, CD, DVD, CD-Rom, jeux vidéo, expositions - on lira à propos de celle qui vient de se terminer à Paris l'encadré des pages 36-37 -, téléfilms d'animation, jeux de rôles, pièces radiophoniques, jouets, etc. $\mathrm{Si}$, à mon avis, les films de la série tendent à instaurer un rapport passablement déceptif avec le spectateur cinéphile comparativement aux standards des productions hollywoodiennes, ce sentiment résulte partiellement de la disproportion existant entre les possibles engendrés par cet univers et la nécessité de n'en actualiser qu'une infime partie dans les treize heures et demie que dure l'hexalogie filmique.

\section{Arrière-plan idéologique}

Incarnation par excellence de "l'industrie culturelle» dont Adorno et Horkheimer stigmatisaient les effets néfastes ${ }^{5}$, les Star Wars ne sont pas sans poser problème sur le plan de l'idéologie dont ils procèdent et qu'ils véhiculent. Dans les années 1980, Robin Wood a examiné en quoi les trois films de Lucas provoquent sur les spectateurs un effet rassurant, «anesthésiant», en reconduisant l'idéologie capitaliste et patriarcale qui caractérise une société américaine pourtant en proie à des crises dont ces films évacuent toute trace ${ }^{6}$. La mise en évidence de la dimension réactionnaire de la première trilogie constitue l'un des lieux communs de sa réception critique 7 . Elle est même en quelque sorte revendiquée par les représentants "officiels» de Lucasfilm pour motiver en quoi Un nouvel espoir (1977) aurait instauré une rupture - qu'il s'agit bien de comprendre ici au sens strictement inverse des "pratiques de rupture" prônées par un cinéma politisé - en proposant un univers de pure fantaisie à une époque où la société était plongée dans le marasme qui succéda au Vietnam et au Watergate (voir par exemple l'introduction du film documentaire L'Empire des rếves. L'Histoire de la trilogie Star Wars $\mathbf{8})$. 
Les remarques des critiques quant à l'idéologie conservatrice des films de Lucas n'oblitèrent cependant pas d'autres axes d'analyse des représentations qui y sont à l'œuvre. Affirmer la productivité théorique d'un tel corpus ne signifie pas nécessairement adhérer à la vision du monde et du cinéma qu'il propose, mais plutôt examiner la place de ces films (et des diverses pratiques signifiantes qu'ils ont suscitées) parmi les productions audiovisuelles contemporaines. Il faut d'ailleurs noter qu'en convoquant sciemment des stéréotypes du cinéma de genre des années 1930-1940, Lucas entendait précisément conférer à ses films une allure désuète dont découle le cadre idéologique de ses récits. Bien que réalisé à l'ère du numérique, La Menace fantôme (1999) continue de faire référence aux films de l'Age d'or des studios hollywoodiens, voire aux premières décennies de la télévision. On peut bien sûr considérer cette démarche comme un refuge dans la nostalgie, mais la légère distance et la désinvolture avec lesquelles le premier opus de la trilogie fondatrice traitait les motifs narratifs incriminés ainsi que ses personnages 9 tendent, de façon certes retorse, à désamorcer la critique qu'il a suscitée à l'époque. Un paradoxe naît lorsque l'on compare les actions représentées - par exemple la décision prise par un contrebandier désengagé de se ranger du côté de l'Alliance Rebelle pour combattre l'oppresseur (sur le modèle de Rick dans Casablanca) - et l'effet que provoqueraient selon Wood les films de la trilogie, qui enjoindraient le public à «évacuer la responsabilité de devoir changer les choses»10. Ce constat ne s'explique pas seulement parce que Wood sous-estime parfois le contenu narratif proprement dit - il est vrai que les tentatives de restauration d'un ordre réactionnaire sont figurées dans les épisodes IV-VI de façon artificieuse comme un acte progressiste $\mathbf{1 1}_{\mathbf{1}}$, mais aussi parce que, à presque tous égards, les Star Wars présentent l'ambivalence d'un objet de la culture de masse qui, par exemple, occulte paradoxalement sa nature "aliénante» (ou infantilisante) à force de l'exhiber. Certaines études menées dans ce dossier relèveront à plusieurs titres le caractère foncièrement contradictoire des Star Wars $\mathbf{1 2}$. Alors que les approches soumises par principe à des dichotomies simplificatrices (l'art vs l'industrie, la critique idéologique vs l'apologie du capitalisme, etc.) passent à côté d'un tel objet (quand elles ne l'ignorent pas purement et simplement), il nous paraît plus productif de nous pencher sur ses paradoxes.

On peut se demander si l'analyse proposée par Wood dans Hollywood from Vietnam to Reagan s'applique également à la seconde trilogie. A mon avis, les trois épisodes sortis à partir de 1999 montrent plutôt qu'en passant de l'ère (pré)reaganienne à celle de Bush, le manichéisme lénifiant a fait place à l'omniprésence du doute. En raison de références
9 Cette dimension est soulignée de façon récurrente par la presse. Le Péron note par exemple à la sortie d'Un nouvel espoir: "Aussi I'histoire, les personnages, les comédiens, n'ont pas en eux-mêmes une grande importance; [...] D'où cette froideur (qui n'est pas une froideur sèche agressive: plutôt cool que cold) et ce sentiment que tout est déjà joué à l'avance [...]" (Serge Le Péron, op. cit., p. 22). Dans un article favorable au film du $n^{\circ} 197$ de Positif (dont la couverture est un plan moyen de Darth Vader), Robert Benayoun notait également que "Luke Skywalker accepte tout ce qui lui arrive avec un sentiment de familiarité, comme quelqu'un que l'inattendu n'étonnera jamais, un abonné de l'extraordinaire" ("L'horizon retrouvé (La Guerre des étoiles) ", in Positif, n 197, septembre 1977, p. 47).

10 Robin Wood, op. cit., p. 165.

11 Voir à ce propos Douglas Kellner et Michael Ryan, Camera Politica. The Politics and Ideology of Contemporary Hollywood Film, Indiana University Press, Bloomington/Indianapolis, 1988, p. 234. Le discours construit par les intrigues des Star Wars me paraît en fait soumis à un principe auquel la production de ces films obéit à tous les niveaux: il s'agit de faire passer l'ancien pour du nouveau.

12 Notons que George Lucas lui-même relève un paradoxe qu'il inscrit quant à lui dans la construction du paradigme auteuriste de l'indépendance envers les studios: "Je me battais contre le système des sociétés [...] mais je me retrouve maintenant à la tête d'une société. Il y a donc là une certaine ironie car je suis devenu la chose même que j'essayais d'éviter... ce qui est en fait partiellement ce dont il est question dans Star Wars" (entretien pour L'Empire des rêves...). La critique française auteuriste a d'ailleurs intégré ce jugement: Alain Garsault intitule en 1983 son article "Les paradoxes de George Lucas " (Positif, n² 271, septembre 1983, pp. 2-6), titre qui est repris seize ans plus tard par Pierre Berthomieu (Positif, $n^{\circ} 465$, novembre 1999, pp. 64-65). 
13 Blocus imposé de façon éhontée par ce fruit de la concentration économique qu'est la Fédération du Commerce, République justicière qui entre en guerre sans remettre en cause les décisions inadaptées de ses dirigeants, "élection" d'un "chancelier" aux pouvoirs illimités résultant d'une totale manipulation, terrorisme, formation dans le secret d'une armée qui s'avérera dangereuse pour la démocratie, massacre d'un peuple des sables aux allures arabisantes par un chevalier aveuglé par une soif de vengeance, etc.

14 Robin Wood, op. cit., pp. 169-170.

15 L'appartenance des Jedi à la hiérarchie militaire est cohérente avec l'épisode réalisé en 1977 , où cet aspect demeurait néanmoins dans le hors-champ du film: rappelons que dans son message de détresse, la princesse s'adresse à Ben en l'appelant "Général Kenobi ". plus directes (qu'elles soient conscientes ou liées à «l'air du temps») à la politique américaine contemporaine ${ }^{\mathbf{1 3}}$, les intrigues se font en effet moins «escapistes", quand bien même la spectacularité tend quelque peu à rejeter de tels enjeux à l'arrière-plan. Le Mal, incarné par le Seigneur des Sith, ne se trouve désormais plus chez les Autres - dans ce camp opposé dont le montage alterné, la musique et les volets se plaisent à souligner chez Lucas l'altérité menaçante -, mais au cœur de la «République». Le Chancelier sournois, qui ne cesse de répéter combien il est mû par un désir d'instaurer enfin la "paix dans la galaxie» (comme on pourrait le dire du Proche-Orient), orchestre dans l'ombre l'intégralité des événements (y compris peut-être l'idylle entre Anakin et Padmé) dans l'intention de saper progressivement tout principe démocratique. La plupart des actions des héros sont vaines, puisqu'elles conduisent inévitablement au renversement de la démocratie. Le futur bras droit du despote diabolique, dont la première trilogie nous avait présenté in extremis le rachat, est initialement présenté comme une figure messianique (Anakin, en lequel Obi-Wan croit reconnaitre celui que les prophéties ont annoncé, est le fruit d'une «Immaculée Conception»), puis nous apparait dans toute son abomination en massacrant, après les habitants du campement d'autochtones qui avaient séquestré sa mère, l'ensemble des enfants appartenant à l'ordre Jedi - La Revanche des Sith se concluant sur une sorte de diaspora où les croyants, après la destruction du Temple, sont dispersés par l'Empire. La légitimité de l'action militaire tend à être remise en cause beaucoup plus explicitement que dans la première trilogie, que Robin Wood considérait déjà comme imprégnée d'une "crainte [...] que la démocratie capitaliste ne soit pas clairement séparable du fascisme et puisse porter en son sein la potentialité de devenir fasciste, totalitaire, l'égale d'une police d'Etat». Il précisait ensuite à ce propos que "l'aspect le plus positivement intéressant des films de Star Wars [...] semble être [...] leur dramatisation de ce dilemme»14. Or le récit des épisodes II et III exploite pleinement cette situation paradoxale où des maîtres Jedi devenus généraux sont propulsés à la tête d'une armée qui est précisément celle dont disposait l'Empire de la première trilogie 15. Dans La Revanche des Sith, Anakin (Hayden Christensen), construit dans un premier temps comme le principal foyer de l'identification spectatorielle, est d'ailleurs conduit à émettre de sérieux doutes sur la légitimité du pouvoir octroyé aux Jedi (ainsi d'ailleurs que Yoda dans L'Attaque des clones, qui fait remarquer combien les Jedi sont devenus «arrogants»). Même s'il s'avère in fine (au sens quasi eschatologique) qu'Anakin est leurré par le tribun Palpatine (Ian Mc Diarmid), certains arguments avancés suggèrent 
que l'existence de l'ordre des chevaliers s'accorde mal avec le maintien de la démocratie.

Ce vacillement des certitudes, suggéré dans les films, apparaît beaucoup plus ostensiblement dans les récits de l'univers étendu situés à l'époque de la guerre des clones (c'est-à-dire entre les épisodes II et III). La première planche du comic book intitulé, dans sa version française, Dernier combat sur Jabiim $\mathbf{1 6}$ est symptomatique de cette orientation récente que Dominic Arsenault et Bernard Perron décèlent également dans le jeu vidéo Knights of the Old Republic mis sur le marché en 2004. Le prologue de la bande dessinée montre en quatre cases une discussion entre deux soldats indigènes de la planète Jabiim (d'apparence humaine) en guerre contre l'armée de la République qui, à l'affût dans un cratère, évoquent les bruits qui courent sur les atrocités perpétrées par les Jedi, qui "coupent les femmes en deux avec leur sabre laser». Ce renversement du point de vue conduit à assimiler la figure par excellence du «sage» aux fascistes de la première trilogie, et cela d'autant plus que cette ouverture évoque exactement, en en inversant les termes, la situation du combat sur Hoth de L'Empire contre-attaque. En effet, alors que le soldat de la planète Jabiim dit des Jedi qu'ils "montent des monstres géants à quatre pattes", il s'interrompt à la suite d'une secousse provoquée par une escouade de gigantesques quadripodes que le lecteur découvre en "contrechamp» sur la double page suivante. Le cratère occupé par ces deux personnages est d'ailleurs cadré de sorte à évoquer une tranchée semblable à celles dans lesquelles se réfugient les soldats de la Rébellion dans L'Empire contre-attaque, qui subissent l'assaut des mêmes machines effrayantes, cette fois aux mains de l'Empire. Cet exemple révèle combien le "discours" formulé par les trois épisodes les plus récents et leurs dérivés paratextuels n'est pas seulement construit de façon interne à ces films, mais naît implicitement d'un dialogue constant avec la première trilogie.

\section{Une "machine " narrative à l'ère de la culture de masse}

La particularité du film de 1977 réside dans l'aisance avec laquelle il sait faire accepter, huit ans après que l'homme a marché sur la lune, un univers galactique où les humains n'ont pas besoin de casques pour respirer dans l'espace, où les sons des canons laser se font entendre dans le vide interstellaire et où les engins volent à la vitesse de la lumière. Le meltingpot référentiel - on passe de la cantina ${ }^{\mathbf{1 7}}$, repère de chasseurs de primes qu'ont popularisé les westerns tournés au Mexique, à des moments de comédies américaines classiques avec le trio Luke-Han-Leia confiné dans des intérieurs, en passant par des scènes issues de films de pirates $\mathbf{1 8}$
16 Haden Blackman et Brian Ching, "Les Faiseurs de pluie. La bataille de Jabiim", in Clone Wars volume 3, Dernier combat sur Jabiim, Delcourt, Paris, 2004 (version originale: Star Wars Republic, $\mathrm{n}^{\circ}$ 55: The Battle of Jabiim, Dark Horse Comics, 2 juillet 2003).

17 Ce terme consacré pour évoquer l'établissement de l'astroport de Mos Esley renvoie explicitement à l'appellation donnée aux saloons dans certains westerns comme El Dorado (Howard Hawks, 1966). L'expression sibylline mais récurrente dans Star Wars de "Bordure extérieure" ("Outer Rim") participe de cette définition d'un espace en fonction d'un au-delà incertain. Lucas déclare d'ailleurs à propos de son intérêt pour l'espace galactique: "J'ai été tout de suite fasciné de constater qu'on pouvait trouver un remplaçant au western, cette mythologie qui était en train de partir à la dérive. L'un des facteurs les plus importants qui se rapportent à cette mythologie, c'est que tout se passe au-delà des frontières, au-delà des montagnes " (cité in Mary Henderson, Star Wars. La Magie du mythe, Presses de la Cité, Paris, 1998,

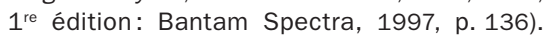
On trouve cette référence dès les premières déclarations du cinéaste, par exemple dans l'entretien qu'il donna à Robert Benayoun et Michel Ciment en 1977: "Pour les gens de ma génération, il n'y a plus d'Ouest. Ce fut la dernière terre exotique, la dernière mythologie" (Positif, n 197 , septembre 1977, p. 53). Pour Robert Luit, qui s'appuie aussi sur les déclarations d'intention de Lucas, "La Guerre des étoiles est un bon film, sans doute le spaceopera définitif, mais c'est avant tout un western de remplacement" ("Les rendez-vous manqués de la S.F.", in Positif, $n^{\circ}$ 263, 1983, p. 3).

18 Un nouvel espoir est véritablement hanté par le souvenir du rythme alerte de L'Aigle des mers (The Sea Hawk, Michael Curtiz, 1940), film dont la musique exercera par ailleurs une influence indéniable sur le travail du compositeur John Williams pour l'ensemble des Star Wars (voir l'article de Laurent Guido dans le présent dossier). De la séquence (quasi) inaugurale de l'abordage d'un vaisseau sur lequel voyage un ambassadeur à la cérémonie présidée par la reine en l'honneur du héros dans un bref finale abruptement clos par le générique, le film de Curtiz présente d'évidentes similitudes avec le premier épisode de la saga de Lucas. 
19 Le rapport de l'élève au maître rappelle bien sûr les intrigues mettant en scène des samouraïs (où l'homme en formation suit les enseignements du "sensei "), et l'attitude prônée celle du "zen". De façon plus anecdotique, le terme de " Jedi " qui apparaît dès les premières versions du synopsis de Lucas est quasi homophone du préfixe de "jidai-geki", appellation qui désigne au Japon les films d'époque comme Les Sept samouraïs (voir Max Tessier, Le Cinéma japonais, Nathan, Paris, p. 107). Quant au nom du maître Jedi Yoda, il fait penser à celui du fidèle scénariste de Mizoguchi, Yoshikata Yoda.

20 Dans la logique de Robin Wood, on pourrait dire également que ce monde fantaisiste n'oppose aucune résistance à son assimilation immédiate dans la mesure où le film reconduit les valeurs (patriarcales, capitalistes, etc.) de la société qui conditionnent par ailleurs le public.

$\mathbf{2 1}$ Si la mention des lieux de tournage relève de l'information paratextuelle, on peut dire que ces trois environnements correspondent néanmoins à des types de paysages immédiatement reconnaissables comme appartenant à notre monde.

22 Sur la notion d'“invariant" au cinéma, voir mon ouvrage La Fiction au cinéma, L'Harmattan, Paris, pp. 144-147.

23 Lorsque Luke Skywalker demande à l'ermite qu'il appelle "le vieux Ben" s'il n'est pas "Obi-Wan", Ben acquiesce en disant qu'«il y a bien longtemps " qu'on ne l'a plus nommé ainsi. Grâce à la musique qui fait retentir à cet instant le thème de Ben (identique à celui de la Force), la réaction du vieil homme prend une dimension particulière, faisant d'autant plus resurgir tacitement le temps des épopées mythiques que la même expression "Il y a bien longtemps" a donné naissance au film dans le carton inaugural (unique mention verbale liminaire, puisque le film, comme tous ceux de l'hexalogie, ne comprend pas de générique initial).

24 The Hero with a Thousand Faces (1949). Cet ouvrage dans lequel le mythologue examine dans une démarche anthropologique un archétype du héros qui s'avère commun à des productions discursives apparemment hétérogènes a connu un succès considérable dans le domaine anglophone (il s'est vendu à plus d'un million d'exemplaires).

25 D'ailleurs, les liens mêmes entre Star Wars et les récits médiévaux font aujourd'hui partie intégrante de la culture populaire, ainsi qu'en témoigne, en France, la récente série télévisuelle Kaamelott d'Alexandre Astier produite par M6. ou par des combats au sabre inspirés du cinéma japonais ${ }^{\mathbf{1 9}}$, ainsi que des batailles "aériennes" analogues à celles des films de guerre - rend les termes du contrat de lecture à la fois éclectiques et parfaitement assimilables à partir d'un certain habitus cinéphilique ou télévisuel 20. Car en dépit des vaisseaux interstellaires dont elle regorge, la saga extrapole fort peu sur les connaissances scientifiques qui lui sont contemporaines. Par exemple, les environnements de la première trilogie n'héritent en rien de la représentation traditionnelle inspirée du sol lunaire, mais sont constitués de paysages qui, quoique passablement hostiles, s'avèrent bien terrestres (le désert tunisien pour l'épisode IV, les glaces norvégiennes pour le V et l'étendue boisée de Californie pour le VI) ${ }^{21}$. En fait, le monde de Star Wars diffère extrêmement peu du monde d'expérience des spectateurs: en "logique des mondes possibles", on dirait que le nombre important d'invariants assure des conditions d'accès très aisées à cet univers ${ }^{22}$. Ainsi, après l'ouverture "spatiale» d'Un nouvel espoir, on «revient sur terre» (ou plutôt sur Tatooine) avec le quotidien d'un jeune paysan rêveur dont les aspirations sont intégralement calquées sur celles d'un adolescent des années 1970.

Significativement, chacun des films débute par la mention "Il y a bien longtemps, dans une galaxie lointaine...", entame similaire au "Il était une fois..." des contes. L'adverbe temporel ("a long time ago»), qui apparaît même au début du film de 1977 dans la bouche de Ben ObiWan Kenobi (Alec Guinness) lorsque ce dernier se réfère à son propre passé (qui donnera à son tour lieu au récit de la seconde trilogie) ${ }^{23}$, s'oppose explicitement au futur des récits d'anticipation pour nous plonger dans le passé des mythes fondateurs, sur lesquels Lucas s'est indubitablement basé pour construire son récit en ramenant diverses sources à des archétypes. D’ailleurs, le cinéaste a toujours souligné l'influence qu'a exercée l'ouvrage Les Héros sont éternels de Joseph Campbell $\mathbf{2 4}$ sur l'écriture de la dernière mouture de son scénario, influence qui est notamment perceptible au niveau de la structure du récit initiatique de Luke. Ainsi, les motifs utilisés sont universellement compris, et susceptibles d'avoir un ancrage dans la société réelle (des spectateurs) et fictionnelle (des personnages). Dans un article du présent dossier où il discute la question des emprunts faits par Lucas à la littérature médiévale, Alain Corbellari problématise, en plus de repérer nombre de parallèles entre certains récits du XII ${ }^{e}$ siècle et Star Wars, le statut même de ces rapprochements dont il s'agit bien souvent de relativiser la portée: la façon dont Lucas évoque par exemple les récits de chevalerie s'inscrit en fait dans un intertexte beaucoup plus global, lié aux origines de la fiction occidentale 25 . Néanmoins, à des fins de valorisation culturelle de Star 
Wars, les commentateurs s'emploient fréquemment à opérer des rapprochements avec des motifs iconographiques ou narratifs appartenant à la culture légitimée 26. Lorsqu'elles ne sont pas forcées, ces associations résultent d'emprunts qui sont effectués de façon si concertée qu'en faire simplement l'inventaire s'avère finalement peu productif, puisqu'on est amené à reconstituer ce que le cinéaste a lui-même conçu, qui plus est sans rendre compte du positionnement quelque peu désinvolte qu'il affiche à l'égard de ces référents. Car l'amalgame ludique auquel s'adonnent Lucas et ses collaborateurs participe pleinement de la culture de masse du $\mathrm{XX}^{\mathrm{e}}$ siècle, de sorte que les significations originelles sont pour la plupart suspendues (bien plus que "détournées»).

Le succès phénoménal de l'histoire de Star Wars prouve toutefois à lui seul l'efficacité de cette démarche de compilation/réactualisation de motifs mythologiques qu'il n'est pas étonnant de voir ravivée dans les années 1970, époque où, dans la foulée structuraliste, l'anthropologie comme l'analyse des récits connaissent un essor considérable qui conduit notamment à redécouvrir des théoriciens plus anciens comme le folkloriste russe Vladimir Propp, dont La Morphologie du conte (1928) connaît une traduction anglaise en 1968. L'entreprise taxinomique qui
26 Voir par exemple le catalogue d'exposition de Mary Henderson, Star Wars. La Magie du mythe, op. cit. Si la mise en parallèle d'images de Star Wars avec certaines sources iconographiques fonctionne tout à fait lorsqu'il s'agit de Flash Gordon, il paraît infondé de rapprocher (pp. 66-67) notamment un plan tout à fait banal extrait d'une scène de baiser entre Harrison Ford et Carrie Fisher d'une peinture représentant Tristan et Iseut (N. C. Wyeth, 1917).

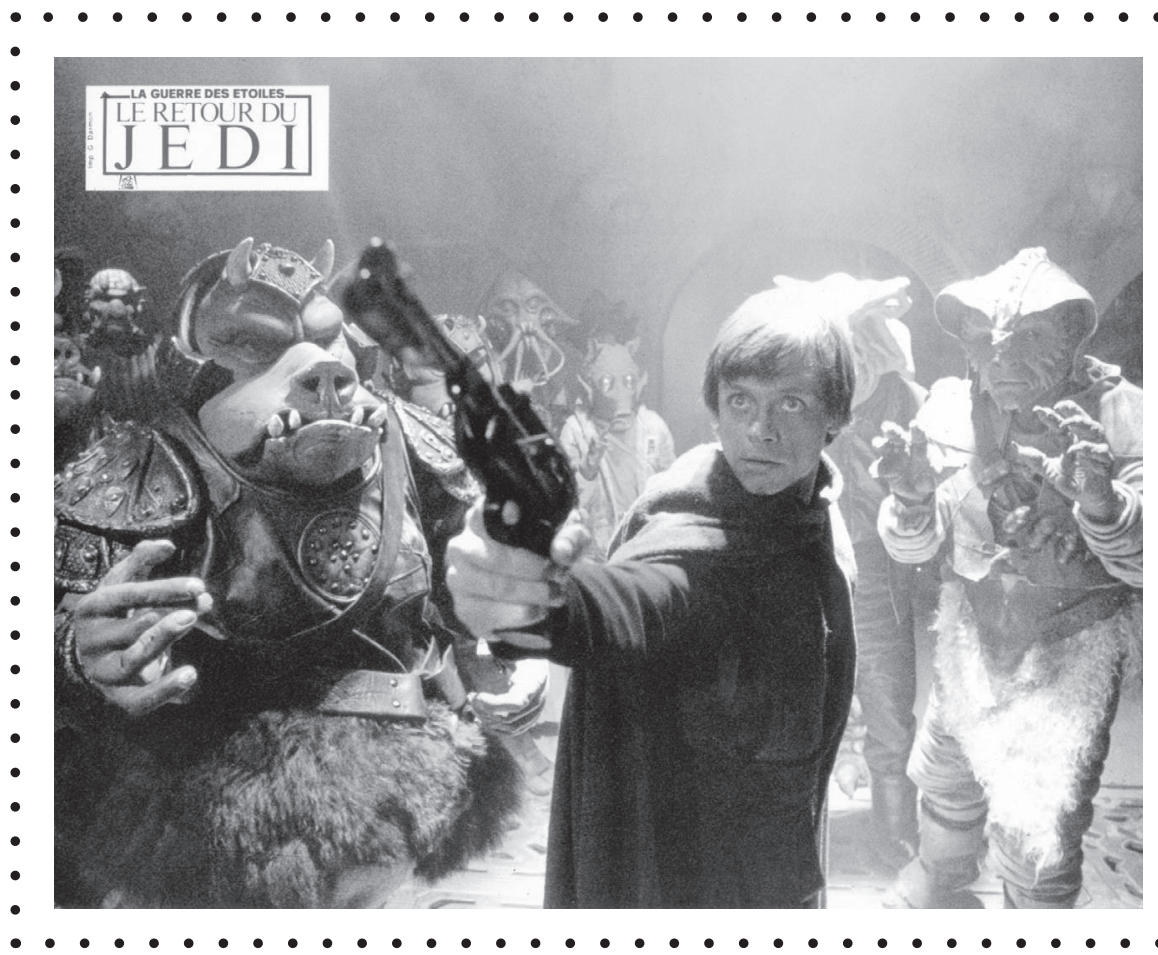

Au centre de l'attention de tous, l'homme blanc (humain et masculin) tenant en respect la horde d'aliens au faciès disgracieux dans cette cour des miracles qu'est le repère du "gangster" Jabba. Cette affiche d'exploitation pour l'Episode $V$ (1983) - image célèbre qui fait par exemple la couverture de l'édition française du roman Le Retour du Jedi (Editions G.P., Paris, 1983) - est révélatrice du rapport à l'Autre qui caractérise la trilogie fondatrice. 
27 Vladimir Propp, Morphologie du conte, Seuil, Paris, 1970, p. 93.

28 II apparaît dès lors rétrospectivement étonnant que ce soit précisément Han Solo, futur amant de Leia (et mari dans l'univers étendu), qui s'adresse à la princesse en l'appelant ironiquement "sister" (voire la séquence dans le cockpit du "Faucon" lors du trajet vers Alderaan), alors que le jeune Luke s'est en fait épris de sa sœur. II est décidément difficile de "Sortir de la famille" dans Star Wars, qui essaie de concilier les motivations amoureuses avec une quasi asexualité des protagonistes. consiste à relever un nombre fini d'éléments constants au sein d'un vaste ensemble de récits s'appuie sur une opération effective de décomposition en unités minimales et sur une recomposition virtuelle à travers les diverses combinaisons desdites unités. Or les auteurs de productions narratives peuvent exploiter à leur tour ce potentiel combinatoire en actualisant certains possibles; c'est dans cet esprit que le scénario-puzzle de Star Wars fut élaboré.

Je ne postule bien sûr pas une influence directe d'un théoricien comme Propp sur la réalisation des scénarios de Star Wars, mais il me semble que l'esprit de ses recherches est caractéristique de la façon dont on considère les questions du récit dans les années 1970. Par exemple, Propp évoque comment la quête du héros se met en place à partir d'une "prise de conscience du manque» - phase que Campbell qualifie d'«appel de l'aventure» - en des termes fort évocateurs si on les rapporte à l'épisode IV de la série Star Wars:

«L'objet du manque peut se faire connaître malgré lui, en se montrant un instant, en laissant derrière lui une trace éclatante, ou en apparaissant au héros sous l'aspect d'une certaine image (portrait, récit). Le héros [...] sombre dans la mélancolie et l'ardent désir de revoir la beauté entrevue.» $\mathbf{2 7}$

Cette description, que le folkloriste rapporte à la structure d'une majorité des contes de son corpus, correspond en tous points à l'apparition «hologrammatique» de la justement nommée "princesse» dans Un nouvel espoir, vision fugace et involontaire (c'est lorsque le jeune homme nettoie le droïde que la projection se déclenche de manière impromptue) que le robot R2-D2 se refuse à diffuser dans son intégralité, en dépit des injonctions pressantes du jeune Luke Skywalker qui s'écrie immédiatement et de façon ingénue "She's beautiful!». La répétition en boucle du même fragment de message confère en outre à cette image un caractère obsessionnel et fétichiste (il constitue véritablement un «objet partiel» au sens freudien). Luke ne verra ce message dans son intégralité que plus tard chez l'ermite Obi-Wan, son futur mentor dont le nom est précisément prononcé dans la séquence où naît la "prise de conscience du manque». Inconsciemment, la vision de cette image tremblante met le héros sur le chemin de sa quête, sans pour autant, il est vrai, que l'on puisse déceler dans le jeu assez sommaire de Mark Hamill une quelconque "mélancolie». Dans ce premier épisode où ne pèse pas encore le spectre de l'inceste $\mathbf{2 8}$, l'image projetée de la princesse est un facteur décisif pour motiver l'abandon par Luke de sa vie de fermier pour devenir un chevalier. C'est d'ailleurs lui qui incitera ensuite Han Solo (Harrison Ford) à libérer Leia (Carrie Fisher) du «labyrinthe» de l'Etoile 
de la Mort («Death Star»), forteresse spatiale dont elle est captive. Cet acte héroïque permet en fait d'articuler l'intrigue individuelle (la quête du Jedi) avec des enjeux collectifs (l'avenir de l'Alliance Rebelle). On le voit, ces motifs sont loin d'être propres à l'univers Star Wars, en dépit de leur apparence spécifique dans la saga.

"Créateur» d'un récit mondialement connu, George Lucas demeure avant tout une personnalité d'importance en tant qu'initiateur de développements technologiques (et d'institutions chargées de les exploiter) qui furent décisifs pour l'industrie du cinéma. Suite au succès commercial de son second long métrage American Graffiti (1973), Lucas devient producteur en érigeant la société Lucasfilm Limited (Ltd.), L’Empire contre-attaque étant ainsi autoproduit à hauteur de 25 millions de dollars ; en 1975, à partir d'une équipe d'artistes et d'ingénieurs créatifs rassemblés pour résoudre les nombreux problèmes soulevés par la réalisation du projet Star Wars (notamment en développant le système du motion control), il crée Industrial Light \& Magic (ILM), département qui, concentré dès 1978 en Californie du Nord, deviendra le studio de special effects le plus important de l'histoire du cinéma, et façonnera significativement l'apparence visuelle des superproductions hollywoodiennes des années 1980 (E.T., Poltergeist, la série des Indiana Jones, des Star Trek et des Retour vers le futur, etc.) en proposant quantité de perfectionnements ou d'innovations techniques, à l'instar du montage virtuel (l'«Editdroid" en 1984) et de l'animation par ordinateur dont se chargera la Lucas Film Computer Division. Or, en 1986, cette dernière s'autonomisera pour devenir Pixar, la célèbre société spécialisée dans le cinéma d'animation qui, en mai 2006, est intégrée à Disney. Aujourd'hui, ILM est à la pointe de l'imagerie numérique, et rares sont les films américains à effets spéciaux et à gros budget qui, à l'instar de certains jalons comme Terminator II (1991), Jurassic Parc (1993), Titanic (1997) ou Hulk (2003)29, ne passent pas en phase de post-production sur les écrans des professionnels de ce studio dont la seconde trilogie Star Wars constitue une véritable vitrine - notons qu'environ 95\% des plans de La Menace fantôme (1999) ont été générés informatiquement ${ }^{30}$. En outre, Lucas se présente publiquement comme un fervent partisan du tournage en HD, technologie qu'il aimerait voir gagner également toutes les salles de cinéma du globe. La même expansion concerne d'autres champs de la technologie audiovisuelle: on peut penser aux jeux vidéo et à LucasArts, dont la franchise la plus lucrative est bien sûr composée de l'univers Star Wars (voir dans le présent dossier l'article de Dominic Arsenault et de Bernard Perron, qui présentent un historique de cet empire vidéoludique et de ses productions), mais aussi, pour la seule composante acoustique, au système multipistes THX
29 Le film d'Ang Lee n'a certes pas été reconnu comme un film-clé en termes d'innovation technologique, mais d'une part il est utilisé par ILM comme une vitrine du studio (voir l'affiche publicitaire "Dream big" sur laquelle le petit Yoda regarde le géant vert Hulk), d'autre part il procède à mon sens d'une exploitation stylistique des effets spéciaux jusqu'à présent fort rare dans ce type de productions (voir mon article: "Style et intermédialité dans Hulk: le split screen, la planche des comics et l'écran d'ordinateur", in Film Style / Cinema and Contemporary Visual Arts, Forum, Udine, à paraître).

30 Selon Lev Manovich, The Language of New Media, MIT Press, Cambridge, 2001, p. 303. 


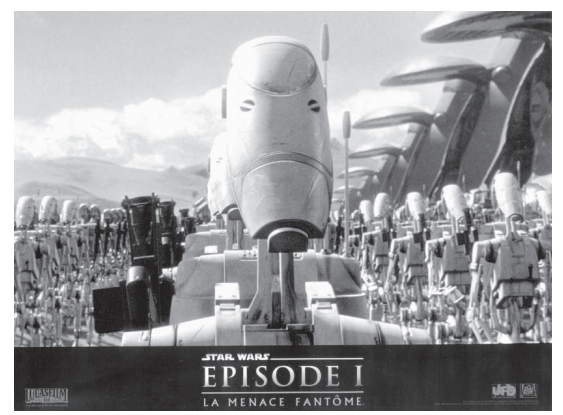

1

31 Ces deux articles sont cités in Laurent Jullier, Star Wars. Anatomie d'un mythe, op. cit., p. 163.

32 Pour cette transposition du couple des compères de La Forteresse cachée (1958) d'Akira Kurosawa, Lucas choisit en effet de vrais acteurs dissimulés dans des costumes. Ainsi même la "boîte de conserve" R2-D2 est-elle animée de l'intérieur par un acteur nain (Kenny Baker) et, lorsque le droïde émet un "bip", le son postsynchronisé est également le produit d'un mélange de mécanique et d'humain, puisqu'il résulte à la fois d'un synthétiseur et de la voix du sound designer Ben Burtt (selon les dires de ce dernier rapportés dans le film L'Empire des rêves. L'Histoire de la trilogie Star Wars). Notons que Burtt continuera de collaborer pour Lucasfilm Ltd. sur la seconde trilogie, marquant l'ensemble de l'univers Star Wars de ses créations sonores. II est également l'auteur d'un moyen métrage documentaire, Effets spéciaux (Special Effects, 1996), qui fut projeté à Paris sur écran géant dans la Géode de la Cité des Sciences, parallèlement à l'exposition Star Wars (voir l'encadré pp. 36-37 de cet article). de fixation et de diffusion des sons qui a su s'imposer mondialement comme un label de qualité, tant dans les salles que pour le home cinema.

Les multiples rapports de ce "cinéaste» (et les guillemets se comprennent aisément au vu de cet éclatement des domaines d'activité de la firme à laquelle est associé son nom) à la dimension technico-industrielle exigent à mon sens d'interroger la façon dont celle-ci est représentée dans la série des Star Wars. N'existe-t-il pas certaines analogies entre l'industrie des produits dérivés en tous genres estampillés «Lucasfilm» et l'armée des robots de la Fédération du Commerce (La Menace fantôme, fig. 1) ou celle des clones (L'Attaque des clones, fig. 2-3) qui, produits à la chaîne, constituent les futurs instruments des forces hostiles à la République que défendent les héros de Star Wars? En ce sens, le récit de ces films est profondément schizophrénique, puisqu'il résulte d'une tentative de concilier le fétichisme de la technologie et la foi en l'humain. Tandis que des instances fictionnelles comme la Fédération du Commerce ou l'Empire assoient leur pouvoir sur leur puissance technologique (le désir d'une "arme absolue» traverse presque tous les épisodes, et trouve son équivalent mystique et positivé dans la «Force»), les réalisations de Lucas ont souvent été accueillies par la critique comme un produit sans âme généré par des machines. Dans Libération, on titrait par exemple à la sortie du Retour $d u$ Jedi «Lucasfilm, la machine à fabriquer des monstres", et on estimait de même en 1999 que "la machinerie Lucas tourne à fond» $\mathbf{3 1}$. Le fait que, dès le premier épisode, deux des rôles principaux du film soient tenus par des robots - certes passablement humanisés 32 - est révélateur de la place accordée par Lucas à la machine. Cependant, comme le note Joseph Campbell (qui commente Star Wars après en avoir involontairement influencé l'élaboration, nouvel exemple d'une exégèse qui se mord la queue), le film de Lucas de 1977 est marquant par le fait qu'il suggère la menace de la technologie et prône la confiance dans les capacités humaines. Ainsi la voix d'outre-tombe suggère-t-elle mentalement à Luke à la fin d'Un nouvel espoir de renoncer à son viseur électronique et de se fier à son instinct pour effectuer son geste salvateur.

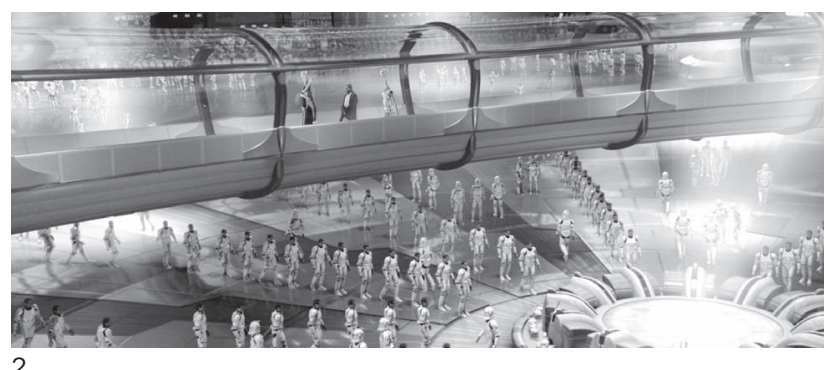

2

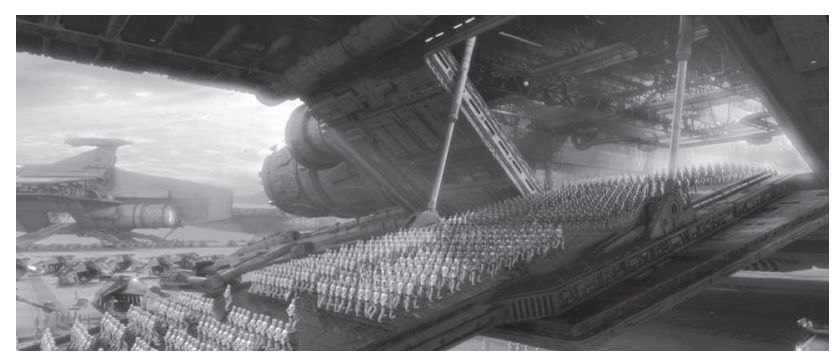

3 
Autre exemple, celui-là mentionné par Campbell: le final du Retour du Jedi où, lorsque "Luke enlève le masque de son père, il abroge le rôle machinique que son père avait joué»33. Ces deux séquences se font certes écho dans leur portée métaphorique - il s'agit en fait de convoquer l'antienne de l'opposition matériel vs spirituel dans un esprit judéo-chrétien de valorisation du second terme, tout en prenant en compte le contexte de l'ère industrielle qui transforme en profondeur cette dichotomie -, mais la double trilogie présente par ailleurs quantité de personnages mécaniques, du côté des «bons» comme des «méchants». D’ailleurs, les films sont indubitablement empreints d'une fascination pour le progrès technologique, fût-il accompli à des fins de destruction. A l'inverse, les machines sont fréquemment anthropomorphisées (notamment sur le plan de leurs manifestations sonores, qui évoquent souvent celles des cartoons) voire entretiennent un rapport d'intimité avec l'individu, tel le "Faucon Millénium», vaisseau du bricoleur fanfaron Han Solo ${ }^{34}$. C'est l'un des principaux paradoxes des Star Wars, et surtout de la seconde trilogie, dont nombre de choix esthétiques dérivent explicitement d'une volonté d'exploiter l'imagerie numérique de façon «transparente» (c'està-dire à des fins d'optimisation du "réalisme» de la représentation), que d'exprimer sur le plan narratif une critique de la technologie tout en véhiculant ce récit grâce à un médium porté à son plus haut point d'élaboration et d'«efficience» technologiques ${ }^{35}$. Comme l'a relevé Laurent Guido, cette contradiction est inhérente à nombre de superproductions contemporaines ${ }^{\mathbf{3 6}}$, mais la représentation du machinique est particulièrement ancrée dans l'imaginaire déployé par les productions cinématographiques affiliées à Lucas. Alors que ce cinéaste substitue sans sourciller des êtres de synthèse à des acteurs en chair et en os, ses scénarios manifestent une crainte certaine devant le devenir-machine de l'homme. On constate que cet élément thématique est présent dès la première version du synopsis de 1973 de Star Wars où, lorsque l'un des derniers Jedi nommé Kane Starkiller se meurt, "tout sauf sa tête et son bras droit [a] été remplacé par des membres et des organes électroniques»37. Cette mécanisation sera le sort d'Anakin Skywalker, réduit à la fin de La Revanche des Sith à un homme-tronc engoncé dans une armure métallique, et menacera son fils Luke, dont la main tranchée lors du premier combat contre son père - perte de l'intégrité corporelle caractéristique des récits initiatiques - sera remplacée par une prothèse dont on le voit activer les servomoteurs. On lira à propos de cette phobie de la mécanisation l'article de Charles-Antoine Courcoux, qui aborde cette problématique sous l'angle particulier (et inédit à propos de Star Wars) de la représentation des rapports sociaux de sexes, principalement dans La Revanche des Sith.
33 Joseph Campbell, The Power of the Myth, Anchor Books, New York, 1991, p. 24.

34 Dans Un nouvel espoir, les ratés successifs du moteur dont se moque la princesse que Han courtise sont autant d'allusions à une impuissance sexuelle momentanée, réactivant l'association entre virilité et véhicule rapide que l'on trouve dans le film précédent de Lucas, American Graffiti.

35 Voir à ce propos mon article "Star Wars, Episode l: la technologie fantôme", in Hors-Champ $n^{\circ} 6$, printemps/été 2001, pp. 36-41.

36 "Plus généralement, on peut percevoir une contradiction fondamentale entre les discours technophobes qui structurent les récits de certains blockbusters [...] et la dimension ultratechnologique de leur facture et de leurs modes de production" ("Entre ccauchemar, et "paranoïa : une introduction aux représentations de la peur dans le cinéma fantastique hollywoodien", in Laurent Guido (éd.), Les Peurs de Hollywood, Antipodes, Lausanne, 2006, p. 38 et note 65).

37 Cité in Marcus Hearn, Le Cinéma de George Lucas, Editions de la Martinière, Paris, 2005, p. 80. Avant la sortie d'Un nouvel espoir, Lucas avouait d'ailleurs: "J'aime regarder les gens vivre avec les machines. Mais mon attitude est ambivalente: je m'en méfie et je les aime. [...] L'homme moderne [...] vit avec ses machines et l'étudier dans cette fonction, c'est faire de l'anthropologie" (Positif, n 197, septembre 1977, p. 58). 


\section{Eviction de l'" auteur", transparence du "style": le primat du récit} et du "monde"

L'univers Star Wars, en tant que représentation visuelle, est né des peintures préalables de Ralph McQuarrie, qui fut par la suite, avec Joe Johnston (devenu plus tard cinéaste), le concepteur d'un grand nombre d'éléments essentiels de la première trilogie, qu'il s'agisse de personnages, de véhicules ou d'environnements. On lui doit notamment, outre Yoda et les quadripodes impériaux, les structures métalliques invraisemblablement ténues (passerelles, antennes, bordures,...) qui, suspendues au-dessus d'un gouffre béant au sein du réacteur central de la station spatiale, supportent Luke lorsqu'il combat Darth Vader au sabre laser dans L'Empire contre-attaque. Sans la force de cette visualisation concrète des idées de Lucas - qui avait néanmoins dès l'origine du projet un réfé-

38 Comme le note Marcus Hearn (op. cit., p. 52), Lucas envisageait initialement de réaliser une adaptation de Flash Gordon, mais les droits ne lui furent pas accordés par le King Feature Syndicate. Le monde créé avec une virtuosité graphique et une inventivité étonnantes par Alex Raymond à partir de 1934 constitue en effet le prédécesseur par excellence de l'esthétique éclectique de la saga de Lucas. Au-delà des volets latéraux et des mentions du générique qui défilent de façon inclinée sur l'écran comme au début de chaque épisode télévisuel de Flash Gordon, Lucas a emprunté quantité de motifs visuels à Raymond, ainsi que ce ton de serial qui confère à Star Wars l'allure d'un film sans prétentions (voir Laurent Jullier, Star Wars. Anatomie d'une saga, op. cit., pp. 97101). Pour la trilogie réalisée récemment, cette référence démarque foncièrement ces films des superproductions contemporaines (l'ouverture in medias res de La Revanche des Sith est symptomatique de ce rythme de comics d'aventure, où il s'agit de rappeler verbalement les péripéties antérieures à chaque nouvelle parution). Notons que peu après Un nouvel espoir, Dino de Laurentiis produit une adaptation de la bande dessinée de Raymond pour le grand écran (Flash Gordon, Mike Hodges, 1980) qui se réfère à son tour à certaines scènes du film de Lucas (notamment lorsque Dale s'enfuit en abattant un garde dans le palais, comme la princesse Leia dans la séquence du vaisseau arraisonné), mais présente une dimension autoréflexive et parodique beaucoup plus marquée. Les motivations ouvertement sexuelles de certains protagonistes (gravitant autour de la Princesse Aura interprétée par Ornella Muti) contrastent en outre avec la pudibonderie des Star Wars. rent iconographique précis, celui de l'univers des comics et des serials de space opera des années 1930 comme Flash Gordon ou Buck Rogers 38 -, la première trilogie se résumerait au contenu somme toute assez élémentaire de son intrigue. Un film ne se fait jamais seul; toutefois, plus que pour tout autre, on peut dire que les Star Wars résultent d'un travail collectif qui s'accommode mal d'une conception auteuriste. D'ailleurs, à l'instar d'autres réalisations cinématographiques de science-fiction de la même époque - contemporaine de l'émergence des revues spécialisées dans le fantastique, vitrines de ce type de productions qui permirent de fidéliser une clientèle -, les premiers épisodes de Star Wars ont popula-

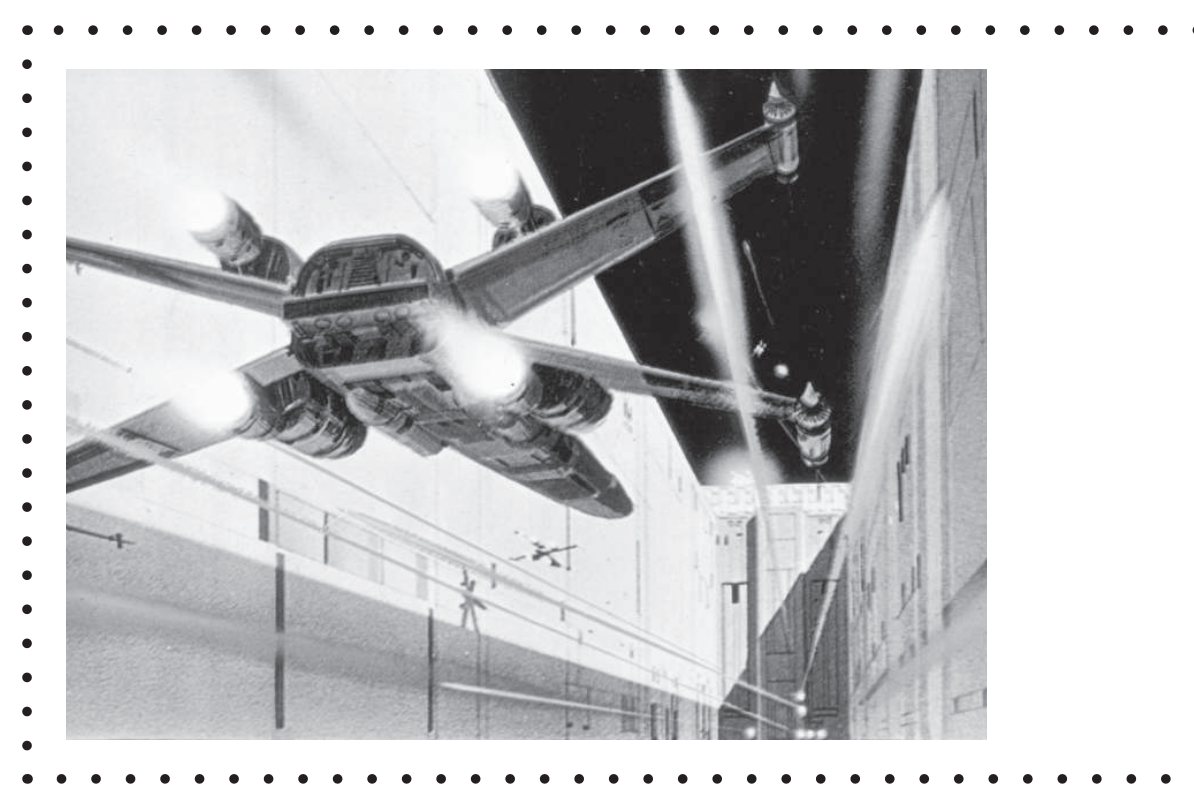


risé l'intérêt pour tout ce qui a trait à la fabrication des films, y compris en ce qui concerne les travaux préalables des designers (dans les ouvrages de type "Art of...").

Bien que George Lucas soit issu d'une génération de cinéastes qui se sont affirmés (c'est-à-dire "construits») en tant qu' "auteurs» (Scorsese, Coppola, De Palma, et même Spielberg qui fait montre d'une productivité étonnante en comparaison de Lucas) ${ }^{39}$, qu'il débute notamment en tant que monteur dans l'équipe de Coppola puis s'illustre dans le domaine de l'«expérimental» et qu'il participe à l'élaboration de films hautement légitimés par la suite (il coécrit notamment une première version du scénario d'Apocalypse Now) ${ }^{\mathbf{4 0}}$, force est de constater qu'il abandonne en 1980 la réalisation pour une période de vingt ans: difficile de faire marcher à la fois le tiroir-caisse et la caméra diront certains (non sans raison), mais cette démarche résulte aussi d'un éclatement de «l'objet-film». Dès lors, Lucasfilm Ltd. devient l'organisme de gestion d'un univers. Le film n'est de ce fait plus une unité close, mais un tremplin, la première pierre d'un édifice aux ramifications multiples. Alors que certains cinéastes ont été érigés au rang des "maudits" du système pour avoir vu leurs films charcutés par les producteurs, Lucas revient sur ses propres films pour les transformer en fonction des possibilités technologiques du moment. L'argument qui apparaît dans toutes les interviews du cinéaste (et que reprend mécaniquement à son compte la majorité des commentateurs) consiste à dire que seule l'image de

39 La relativisation du rôle joué par de tels "indépendants" dans les années 1970 peine à s'imposer dans le domaine francophone. On notera tout de même l'article de Joël Augros dans le dossier récemment consacré par la revue Positif à cette période qui, dans une optique économique, affirme que "la Hollywood Renaissance est un rideau de fumée trompeur" ("Quand on parle du Lew...", in Positif n० 545, juillet-août 2006, p. 13). Certes, Lucas se passe de la Fox pour la production de L'Empire contreattaque, mais cette démarche s'inscrit précisément dans les nouvelles orientations des majors hollywoodiennes qui réaffirment leur pouvoir en "externalisant la production dans des compagnies indépendantes', mais contrôlant le secteur stratégique de la distribution" (id., p. 16).

40 Sur les six années passées par Lucas à rédiger le script de ce film qu'il prévoyait de réaliser en $16 \mathrm{~mm}$ pour Columbia, voir Chris Salewicz, George Lucas. The Making of His Movies, Thunder's Mouth Press, New York, 1999 (1998), pp. 39-41.

Un nouvel espoir (1977) et La Menace fantôme (1999) : la récurrence du travelling avant, figuretype de l'immersion sensorielle du spectateur.

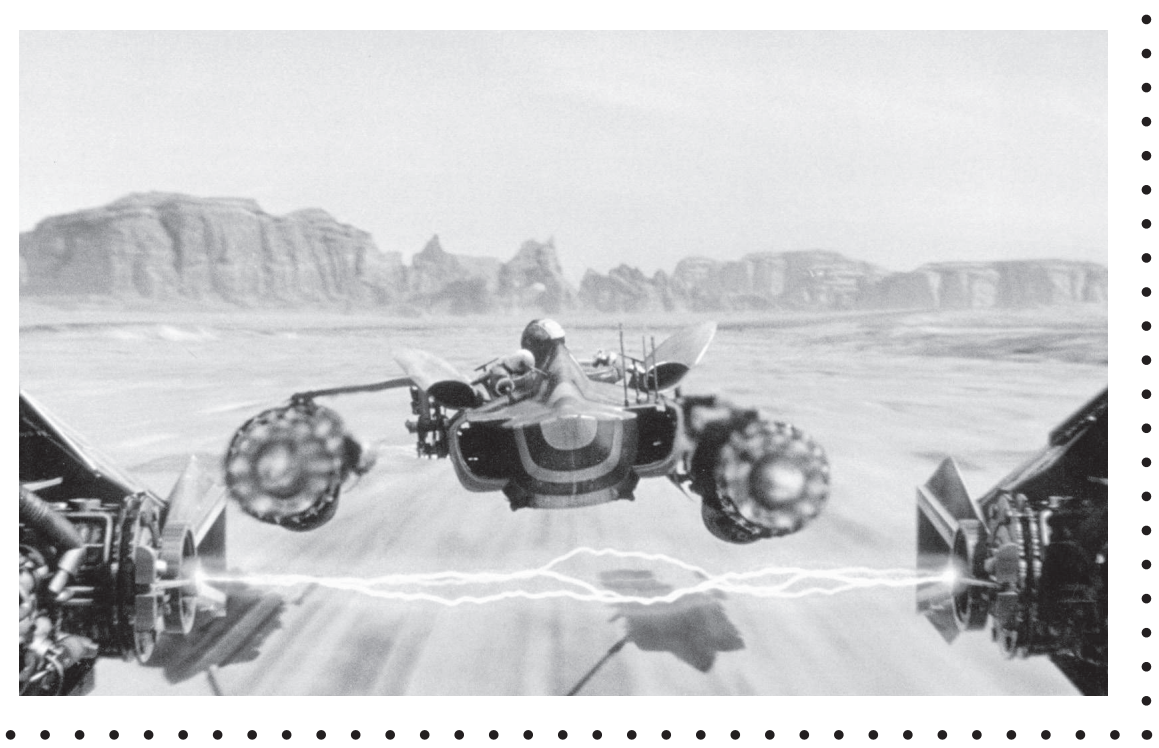


41 Trois DVD doubles comprenant la version "restaurée" et originale de chaque film sont apparus sur le marché en septembre 2006. Ce report de deux ans permet de répéter l'opération commerciale des Special Editions (logique que les éditeurs de DVD connaissent bien): tenter de vendre (presque) la même chose à deux reprises aux mêmes acheteurs. Cette édition ne résulterait cependant pas d'un intérêt subit de Lucas pour les états antérieurs de ses films, mais d'une pression des fans qui souhaitaient cette ressortie et l'exigèrent par l'intermédiaire d'une pétition en ligne.

$\mathbf{4 2}$ Lors de l'apparition sous forme spectrale d'Anakin Skywalker à la fin du Retour du Jedi de 2004 (surimpression qui est à compter parmi les effets au charme désuet des films de Lucas), ce n'est plus Sebastian Shaw qui interprète le personnage (quand bien même il incarne ce dernier lorsqu'il agonise auparavant, une fois retiré le masque qui faisait de lui Darth Vader), mais Hayden Christensen, jeune acteur de la seconde trilogie. Alors que Ben Kenobi, qui apparaît à ses côtés en fantôme, est âgé (il s'agit d'Alec Guinness, non d'Ewan McGregor), Anakin est jeune. Ce fait pourrait paraître paradoxal, mais s'inscrit dans l'interprétation rétrospective de Lucas: le personnage est montré avec l'apparence qu'il possédait avant qu'il ne devienne maléfique. La substitution d'un acteur à l'autre actualise donc le motif de la rédemption, même s'il rompt la logique selon laquelle Luke est placé sous le regard de ses "pères" (Christensen est plus jeune qu'Hamill!). synthèse permet de réaliser aujourd'hui ce qu'il souhaitait obtenir jadis. Dans cette optique, les modifications récentes viendraient actualiser une version originale "virtuelle» que Lucas avait en tête à l'époque, accréditant ainsi la conception selon laquelle il serait un "auteur» inventif qui avait tout prévu dès les premières ébauches, comme si l'univers narratif et visuel de Star Wars ne tenait qu'à une seule personne. Ce work in progress affiche une totale absence de respect pour «l'original»-l'édition DVD de 2004 de la première trilogie, une reprise non exempte de modifications des "Special Editions" sorties en salle en 1997, ne contenait pas les versions originales, dès lors devenues indisponibles durant près de dix ans $\mathbf{4 1}$ - et pour la performance des acteurs (qui n'est certes souvent pas d'un intérêt renversant) au moment du tournage : ainsi un comédien peut-il se voir purement et simplement remplacé par un autre $\mathbf{4 2}$, ou subi-

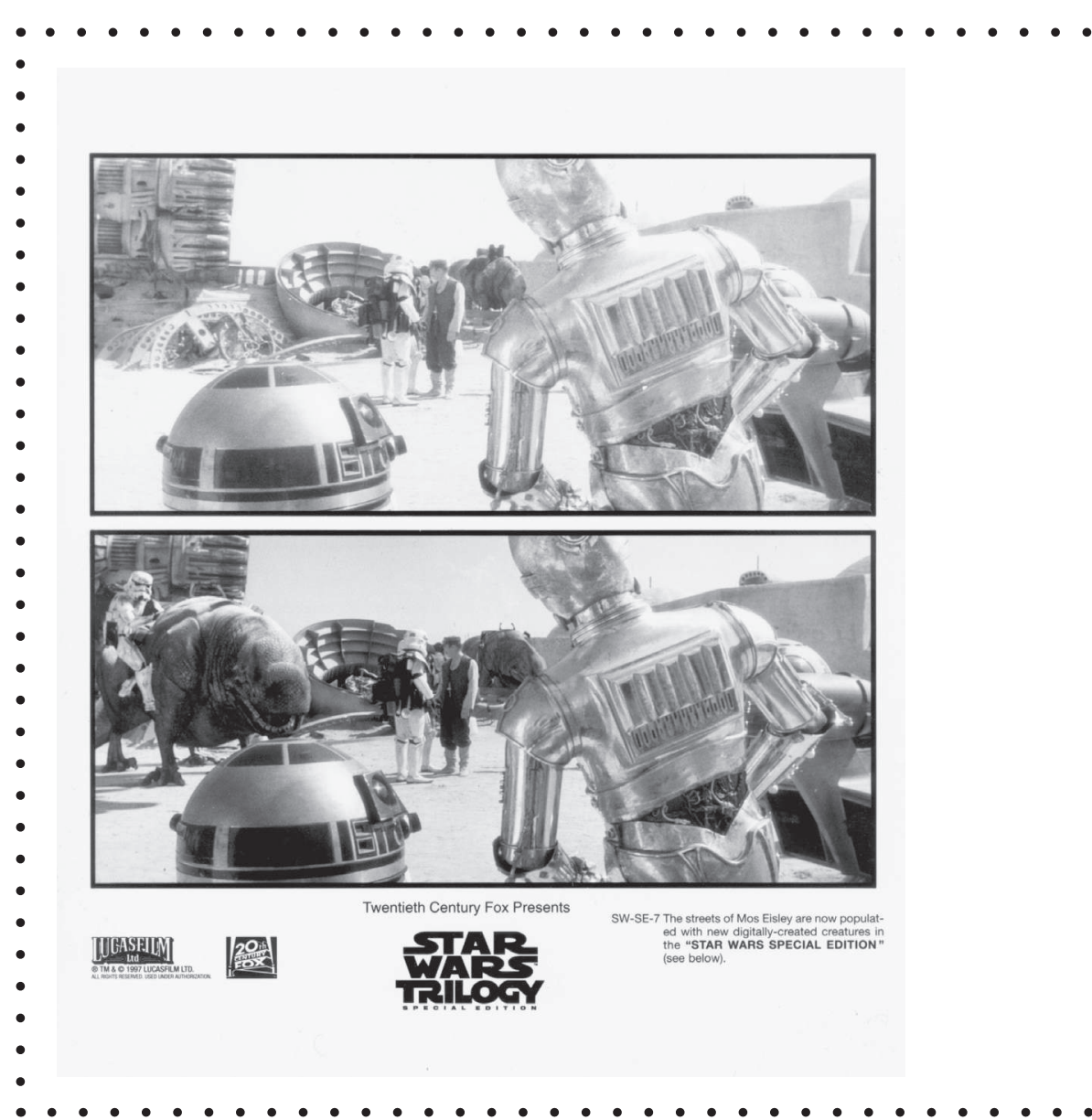


tement affublé d'un mouvement effectué grâce à un effet numérique réalisé plus de vingt après la sortie du film $\mathbf{4 3}$.

Cet irrespect naïvement iconoclaste fait de Lucas l'un des principaux représentants $\mathrm{du}$ "cinéma des attractions» contemporain $\mathbf{4 4}$ : non seulement ses films sont ponctués de moments spectaculaires de bravoure (comme en présentent de nombreuses productions hollywoodiennes), mais l'aspect technologique et les conditions de consommation tendent à constituer une des préoccupations centrales de l'entertainer. Ce qui prime, c'est l'optimisation de l'effet produit sur le spectateur, qui entraîne une "lecture énergétique» (au sens de Roger Odin) paradoxalement réinvestie au profit de l'immersion du spectateur dans le monde du film (contrairement à ce que postule Odin, "l'effet-image» [ne] l'emporte [pas] sur 〈l'effet-monde`», mais fonctionne au bénéfice

\section{Faire (croire que l'on fait) du neuf avec du vieux}

Document promotionnel pour la ressortie de l'épisode réalisé en 1977 dans sa version "Special Edition" vingt ans plus tard. La comparaison révèle l'optimisation de la qualité de l'image au niveau de la luminosité et du contraste. Avant de greffer virtuellement de nouveaux éléments dans l'image, Lucas a dû se soucier du support matériel de son film en faisant restaurer le négatif original.

\section{Autonomie du "plan-tableau "}

Le plan dont sont extraits les deux photogrammes représentés ici, d'une durée de sept secondes intercalées entre un volet latéral et une coupe franche, n'est ni précédé ni suivi d'autres plans qui découperaient l'espace qu'il montre, mais fait office d'insert (équivalent du "pendant ce temps" caractéristique de la bande dessinée d'aventure) à l'intérieur de la séquence de la cantina.

La logique "additionnelle" des interventions infographiques modifie passablement la composition originale de l'image, qui se densifie et multiplie ses centres d'intérêt.
43 La scène d'Un nouvel espoir où le personnage interprété par Harrison Ford abat le précepteur de Jabba en tirant sous la table a été modifiée en 1997 pour faire en sorte que le contrebandier ne tire qu'en situation défensive. Ainsi, dans la seconde version de cette scène filmée en une série de champs/contrechamps frontaux comme dans les westerns-spaghettis (voir l'importance de la botte de Han au premier plan de l'image), ce n'est plus Han Solo mais l'alien qui tire le premier. Pour réaliser ce changement, Lucas a dû faire bouger l'acteur dans l'image (dans le plan d'ensemble final qui comprend les deux personnages) pour signifier que Han esquive le tir, ce qui n'occasionne pas de saute puisqu'un insert d'une explosion intervient au milieu de ce plan. Dans l'édition DVD de 2004, cet effet numérique a été quelque peu amélioré au niveau du respect de la direction des regards (voir, pour l'ensemble des modifications effectuées sur l'épisode, la comparaison des versions proposée sur le site www. starwars-universe.com). II s'agit dans ce cas non pas d'une volonté d'accroître la perfection illusionniste de la représentation (au contraire, elle en pâtit dans la version restaurée pour le grand écran), mais véritablement de s'autocensurer pour des motifs idéologiques (le futur "bon" de l'histoire ne peut être aussi cruel).

44 Suivant la proposition d'André Gaudreault et Tom Gunning, de nombreux historiens et théoriciens du cinéma ont appliqué la notion d'" attractions " (empruntée à Eisenstein) à la période du cinéma dit "des premiers temps" (couvrant environ la période 1895-1908). Philippe Dubois donne une définition synthétique de ce concept qui touche plus généralement à d'autres moments de l'histoire du médium: "le 'cinéma des attractions , c'est celui qui joue la monstration plutôt que la narration, la présentation plutôt que la représentation, la temporalité ponctuelle (hic et nunc) plutôt que l'organisation dans la durée, l'interpellation directe du spectateur plutôt que sa suspension et son conditionnement au suivi d'une diégèse, l'exhibition accrocheuse de ses propres moyens de figuration plutôt que l'effacement en vue d'une transparence de l'action racontée, l'excédent rhétorique plutôt que la norme classique, la recherche de l'événementialité visuelle 'pure, par les formes les plus franches, etc." (Philippe Dubois, "La ligne générale (des machines à l'image)", in Frank Beau, Philippe Dubois, Gérard Leblanc (éd.), Cinéma et dernières technologies, DeBoeck Université, Paris, Bruxelles, 1998, p. 84). 
45 Roger Odin, De la fiction, DeBoeck, Bruxelles, p. 160. Lorsqu'Odin traite d'un "nouveau spectateur" qu'il oppose au "spectateur fictionnalisant" du cinéma classique, il fait invariablement référence à la version de Metropolis de Giorgio Moroder (1984); or l'influence du film de Lang (1927) sur l'univers visuel de Lucas est évidente, ne serait-ce que dans l'apparence du droïde de protocole C-3PO qui ressemble beaucoup au robot censé se substituer à Maria (et ce d'autant plus dans les dessins initiaux prévus pour Star Wars, où le robot apparaît franchement féminisé). En outre, le travail de sonorisation et de colorisation effectué par Moroder n'est pas sans parenté avec les opérations effectuées par Lucas sur ses propres films pour leur ressortie.

46 Laurent Jullier, L'Ecran post-moderne...,op. cit., p. 8.

47 Laurent Jullier, Star Wars..., op. cit., pp. 33-34.

48 André Gaudreault qualifie d' uniponctuels" les "films" des premiers temps qui, participant d'une logique de la "vue", ne comportaient qu'un seul plan (Du littéraire au filmique, Armand Colin / Nota Bene, Paris, 1999, $1^{\text {re }}$ éd. 1988). En proposant cette comparaison, on passe certes de l'image documentaire des bandes Lumière à une représentation hyperfictionnelle, mais c'est la même logique spectaculaire que reconduit le film projeté dans le Star Tour. Bien sûr, les nombreux trucages visuels introduisent une forme de "montage" à un autre niveau (comme les "arrêts de caméra" de Méliès).

49 Voir le site: http://webcot.free.fr/articles/ lucas/startours.htm

$\mathbf{5 0}$ Voir Raymond Fielding, "Hale's Tours: Ultrarealism in the Pre-1910 Motion Picture ", in John Fell (éd.), Film Before Griffith, University of California Press, Berkeley / Los Angeles, 1983, ou plus généralement le $\mathrm{n}^{\circ} 6$ de Décadrages (dossier "Train et cinéma", automne 2005), dont la couverture reproduit une publicité pour le dispositif spectaculaire du Hale's Tour. de ce dernier) 45 . Il ne faut toutefois pas surestimer l'importance du pôle attractionnel dans les films de la saga où le narratif demeure un enjeu central. Laurent Jullier, qui considère pourtant l'épisode de 1977 comme le "coup d'envoi de la réponse du cinéma aux sollicitations postmodernes» $\mathbf{4 6}$, précise fort justement dans son ouvrage récent que les «moments de feu d'artifice se trouvent sertis si profondément à l'intérieur d'une chaîne narrative causale que l'on peut difficilement les prendre pour de véritables mises entre crochets de l'action»47. Le point d'orgue du régime spectatoriel des «attractions» est probablement atteint par le Star Tour, «attraction» au sens littéral du terme que Disneyland consacre depuis 1987 à l'univers Star Wars: le public qui assiste à une projection d'un film de quelques minutes - précisément « uniponctuel ", puisqu'il ne comprend aucune coupe 48 - se trouve dans une sorte de simulateur de vol construit sur un système hydraulique ${ }^{49}$. Toutefois, là non plus, la dimension diégético-narrative n’est pas totalement évacuée: non seulement on situe cette attraction dans la chronologie des films (l'histoire minimale qui y est racontée se situe peu après Le Retour $d u$ Jedi), mais le contexte de Disneyland est lui-même intégré à l'univers du film - comme dans Total Recall (Paul Verhoven, 1990), mais sans la critique larvée de l'aliénation du consommateur -, puisque le "personnage» dont le spectateur prend la place est un client d'une compagnie de voyage intergalactique (la Star Tour Travel). En fait, comme cela était le cas dans le Hale's Tour du début du XXe siècle (1904-1905) où le spectateur contemplait l'écran comme s'il regardait par la fenêtre d'un train en mouvement ${ }^{50}$, le public du Star Tour est convié, dans l'univers diégétique, à regarder à travers le hublot d'un vaisseau spatial. A cet égard, on peut effectivement dire que Star Wars rattache le "cinéma» aux origines attractionnelles du médium, les nouvelles technologies réactivant certains modes de visionnage apparus à l'époque de l'émergence de la culture de masse.

Pour en revenir aux films, il apparaît que ce qui retient l'attention dans la série des Star Wars, ce sont les éléments du monde représenté, rarement par contre la façon dont ils sont filmés. En dépit de la virtuosité affichée sur le plan technologique, ces films requièrent la «transparence» de la forme qui sied à l'optimisation des effets visuels et dramatiques. Il suffit de comparer la facture des Star Wars avec la composition des plans d'un film comme Kagemusha (1980) d'Akira Kurosawa envers lequel Lucas nourrit une sincère admiration (il est d'ailleurs le producteur exécutif de ce film à une époque où le cinéaste japonais ne trouve plus les moyens de produire ses films dans son pays) pour saisir le déficit esthétique de ceux-là. Dans la seconde trilogie réalisée entre 1999 et 2005 
dont Lucas «signe» les trois volets, on constate de façon encore plus flagrante combien la mise en scène est écrasée par l'anticipation des opérations de postproduction. Il n'est dès lors pas étonnant que l'avenir filmique de l'univers Star Wars se «dessine» du côté du cinéma d'animation, dans la mesure où ce dernier parachève l'éviction totale du travail de mise en scène $\mathbf{5 1}$.

S'il fallait trouver au niveau des pratiques filmiques à l'œuvre dans la nouvelle trilogie quelque chose qui la caractérise en propre, on penserait probablement à cette tendance, liée à la nécessité de greffer après le tournage quantité d'éléments dans l'image 52 , à construire des "planstableaux" qui ne sont pas sans évoquer ceux que les historiens du cinéma ont examinés à propos du "cinéma des premiers temps». Après tout, l'autre George(s), Méliès, agençait également le profilmique en fonction des «trucages" prévus pour ses bandes. Sur un plan plus général, on peut se demander si l'intérêt de Lucas pour des images grouillantes de personnages et d'objets mobiles conduit à réactiver l'absence de mise en évidence d'éléments saillants qui caractérise la composition des "films" (souvent composés d'un unique plan) du tout début du $\mathrm{XX}^{\mathrm{e}}$ siècle, où il n'était pas rare qu'une même vue présentât plusieurs actions simultanées. On note d'ailleurs chez Lucas - et plus particulièrement dans La Menace fantôme, épisode auquel ces remarques s'appliquent en priorité - une certaine prédilection pour la figure du raccord dans l'axe (cut-in), qui consiste, avant de tourner le plan suivant, à s'avancer ou à reculer sur la ligne imaginaire reliant l'emplacement de la caméra à l'objet filmé. Conjugué à une profondeur de champ dont l'importance constante sur l'ensemble de l'hexalogie ressortit à un principe qui interdit d'orienter le regard du spectateur par le jeu des zones nettes se démarquant des surfaces plus floues $\mathbf{5 3}$, ce type de raccord permet notamment de conserver la frontalité du cadrage et, dans la perspective d'ILM, de ramener aisément l'espace profilmique à un fond bidimensionnel. Or André Chaperon note à propos d'un film de 1905 que le cut-in constitue l'unique raccord conventionnalisé à l'époque du cinéma des premiers temps, et qu'il diffère d'autres modalités de montage en ce qu'il «circonscrit l'exercice du regard dans un seul et unique espace, composé le plus souvent de manière centripète, sans postulation aucune de horschamp»54. Bien que l'univers de Star Wars soit perpétuellement inscrit dans un mouvement d'expansion (une sorte de hors-champ permanent), il faut convenir que Lucas envisage ces "plans-tableaux» comme des condensations métonymiques de cet univers. Les commentaires des critiques ont montré que cette esthétique ne correspondait aucunement aux canons actuels, la saturation et le "pluricentrage» de l'image étant perçus
51 Ainsi que le déclare George Lucas: "[...] nous avons des idées pour Star Wars. J'ai raconté la saga, c'est-à-dire l'histoire d'Anakin Skywalker. Maintenant, nous allons poursuivre avec un dessin animé 3D pour la télévision. Pour cela, nous travaillons en Asie et construisons des studios à Singapour, et nous ferons de notre mieux pour tirer parti des extraordinaires talents artistiques de la communauté asiatique, du Japon et de I'Inde" (in Lucasfilm Magazine, n 55, septembre-octobre 2005, p. 4). On retrouve là les aspirations initiales de Lucas, qui déclarait en 1977: "Ce que j'aime avec la S.F., c'est de pouvoir créer tout un univers, alors que dans un livre cela reste plus cérébral. [...] C'est un défi très excitant et qui m'a toujours séduit depuis mes premières études de dessin et mon travail dans l'animation à l'Université" (Robert Benayoun et Michel Ciment, "Entretien avec George Lucas ", in Positif, n 197 , septembre 1977, p. 54 ; je souligne).

52 Comme l'a montré Lev Manovich (op. cit., pp. 136-145), les images de synthèse ont suscité une tendance au "non-montage", la spatialisation des informations narratives permettant d'occulter l'extériorité de certaines greffes visuelles par la représentation continue d'une portion donnée d'un univers.

53 Ainsi que le note Laurent Jullier, Star Wars..., op. cit., pp. 41-42.

54 André Chaperon, "Le hors-champ de I'histoire. Une lecture benjaminienne de Tom, Tom, the Piper's Son", in Décadrages n $1-2$, Lausanne, p. 18. 
55 Pierre Berthomieu décrit ainsi La Menace fantôme: "Une action a lieu au premier plan, tandis que de multiples actions peuplent les arrière-plans, comme dans un grand tableau narratif. Au lieu de découper pour préciser les éléments, Lucas enchaîne les plans, la richesse visuelle agissant plus comme sensation globale que dans ses détails, impossibles à appréhender en une seule vision" ("Star Wars Episode I: La Menace fantôme. Les paradoxes de George Lucas ", in Positif, n 465, novembre 1999, p. 65; je souligne).

56 Ainsi Baptiste Piégay fait-il remarquer à propos de La Menace fantôme: "L'art scénographique de Lucas se révèle d'une indigence stupéfiante: il sature l'écran de personnages, de monstres [...], de vaisseaux, de minutieuses explosions, de décors imposants, trahissant une peur du vide assez naïve - et pourquoi pas touchante - mais se révèle paradoxalement incapable d'organiser l'espace " (Cahiers du cinéma, $n^{\circ} 539$, pp. 81-82).

57 Sur les rapports entre diégèse et récit, voir Dominique Chateau, "Diégèse et énonciation ", in Communications, $n^{\circ} 38$, Paris, 1983, et le chapitre 6 de mon ouvrage Du bonimenteur à la voix-over. Voix-attraction et voix-narration au cinéma, Payot, Lausanne (à paraître).

58 Laurent Jullier, Star Wars..., op. cit., p. 41; voir aussi L'Ecran post-moderne. Un cinéma de l'allusion et du feu d'artifice, L'Harmattan, Paris, 1997, pp. 80-84. soit comme des entraves à la lisibilité55, soit comme les symptômes d'une incapacité à représenter l'espace (en postulant bien sûr les normes qui se sont institutionnalisées après le "cinéma des premiers temps")56.

D'importantes différences existent toutefois par rapport au cinéma des premiers temps qui limitent sérieusement la portée du rapprochement: d'une part ces plans, d'ailleurs fort brefs en regard de la durée totale du film, ne possèdent pas d'autonomie narrative, car ils ne relèvent jamais purement du pôle attractionnel; d'autre part, le mixage-son supplée en grande partie la fonction d'orientation de la perception visuelle qu'endosserait un mode de composition centrifuge. Ce que révèlent par contre ces plans généraux denses en éléments susceptibles de capter l'attention, c'est un primat accordé à l'espace sur le temps. Alors que, dans la majorité des films, le diégétique est totalement soumis à sa fonctionnalité narrative, dans Star Wars le monde tend à s'émanciper de la nécessité de raconter une histoire 57 . C'est pourquoi nous avons demandé aux illustrateurs des deux séries d'images que comporte notre dossier, Léonard Félix et Pietro Maffei, de s’inspirer librement d'un environnement spécifique de l'hexalogie (le puits de ventilation de L'Empire contre-attaque pour l'un, Tatooine pour l'autre), cette fois libéré de son asservissement aux actions diégétiques, et même aux films. Nous sommes néanmoins bien loin, dans la saga produite par Lucas, de l'évacuation du récit traditionnel comme le prônaient par exemple les écrivains du Nouveau Roman: au contraire, cette autonomisation de la diégèse est la promesse d'autres récits. L'univers filmique contient en luimême l'amorce d'un "univers étendu».

L'autre trait stylistique des Star Wars repose sur une pratique filmique opposée à la fixité du "plan-tableau ", mais qui obéit au même principe d'une découverte progressive d'un monde: il s'agit de ce que Laurent Jullier nomme le «travelling avant immersif»58, où le spectateur occupe la place d'un pilote - sa place effective aux commandes de l'appareil, ou, par "déplacement», une position située derrière l'engin - dont le véhicule file à vive allure dans un environnement favorable à la mise en évidence de la construction fortement perspectiviste de l'image (couloir, boyau, rangées d'arbres dont les lignes convergent au point de fuite). $\mathrm{Ce}$ type de situation que l'on trouve dans la bataille finale du film de 1977 fait immanquablement penser aux jeux vidéo (ou plutôt à ce qu'ils sont devenus plus tard); d'ailleurs les concepteurs de LucasArts ne se sont pas privés d'exploiter une esthétique qui appelait véritablement l'interactivité dont dispose leur médium. Le fait que ce type d'environnement soit favorable à une représentation infographique est en quelque sorte mis en abyme dans Un nouvel espoir, puisque les représentations schématiques 
du lieu de la bataille projetées sur les écrans lors du briefing des Rebelles constituaient précisément "l'une des toutes premières applications de l'image de synthèse au cinéma» $\mathbf{5 9}$.

Y a-t-il d'autres aspects de la facture proprement dite des Star Wars qui paraissent récurrents et saillants? Il faut à cet égard souligner leur ton particulier, qui tient autant à la référence à la forme feuilletonesque, à la musique si caractéristique de John Williams qu'à l'univers sonore en général, ce dernier devant probablement beaucoup à la collaboration initiale de Lucas sur THX 1138 (1971) avec celui qui deviendra l'un des plus célèbres sound designers, Walter Murch, dont il fit la connaissance à l'Ecole de cinéma de l'Université de Californie (USC). Dans une large mesure, la singularité du film de 1977 résidait à cette époque dans la rencontre - à nouveau inscrite dans le tiraillement entre l'humain et la machine (et corrélativement entre la citation d'un cinéma antérieur et une tentative de renouvellement) - entre, d'une part, une musique instrumentale située aux antipodes des compositions pour synthétiseurs et, d'autre part, une variété proprement "inouïe» d'effets sonores bigh tech inventifs, tant en ce qui concerne les bruits (explosions, tirs des "blasters", ronronnement des moteurs, vrombissements des sabres laser brisant l'air ou se frappant, etc.) que les voix (souffle de Darth Vader, rugissements de Chewbacca, timbre des robots, etc.). Laurent Guido examine dans ce dossier l'inscription du travail du compositeur John Williams dans la tradition wagnérienne, tout en soulignant l'importance d'un héritage plus fondamentalement ancré dans les pratiques du cinéma hollywoodien classique. A travers une réflexion sur la musique de Star Wars, c'est tout l'intertexte généré par ces films qui est convoqué.

\section{D'une trilogie l'autre: temps diégétique et vécu des spectateurs}

Le fait de réaliser à partir de la fin des années 1990 une série de films qui constituent la prélogie de la série fondatrice est à mon sens un "geste» dont les conséquences sur la lecture que certains spectateurs font de l'ensemble de l'intrigue filmique des Star Wars me paraissent essentielles. Il est vrai qu'à partir de sa ressortie en 198160, le film de 1977 comportait au générique la mention "Episode IV: Un nouvel espoir» qui appelait le récit des aventures antérieures. En choisissant toutefois de ne pas baser ses dernières réalisations sur les épisodes VII-IX, Lucas optait pour un récit passablement déterministe: la seconde trilogie devait déboucher tragiquement sur l'avènement de l'Empire et sur la disparition quasi totale de l'ordre des Jedi, rejoignant une noirceur qui caractérise uniquement (et dans une moindre mesure) le «deuxième acte» de la première trilogie (L'Empire contre-attaque).
59 John Knoll, "Jour n 34 ", Star Wars. 365 jours, Editions de la Martinière, Paris, 2005.

60 Les monographies sur George Lucas précisent invariablement que la première trilogie était prête sur le papier avant le tournage d'Un nouvel espoir. Cette affirmation est à la fois juste et fausse: il est vrai que le cinéaste envisageait une trilogie, mais cela ne signifie pas que le scénario des épisodes $V$ et $\mathrm{VI}$ existait déjà. Ainsi, le producteur Howard Kazanjian dit à propos de la genèse de L'Empire contreattaque: "On se réunissait à Medway, qui était le bureau de George à San Francisco, et on regardait les illustrations pendant que George écrivait le scénario" (entretien in L'Empire des rêves. L'Histoire de la trilogie Star Wars). 
$\mathbf{6 1}$ Celui des "mondes" de la nouvelle trilogie qui continue partiellement de s'inscrire dans le paradigme du "mécanique délabré " est justement la planète Tatooine de l'épisode I (dépôt du ferrailleur Watto, atelier d'Anakin à domicile) que l'on trouvait "déjà" au début d'Un nouvel espoir et du Retour du Jedi. L'exception est également le personnage de C-3PO, robot que l'on voit progressivement évoluer dans l'ordre de la chronologie diégétique (ses entrailles électriques sont entièrement visibles dans l'épisode I, à la fin duquel il obtient une carrosserie grisâtre; elle sera ensuite dorée).

62 De nombreux bruits de la première trilogie sont toutefois repris ou déclinés dans la plus récente, si bien que l'univers sonore constitue un facteur décisif de cohésion du monde Star Wars, ainsi que le démontrent également les dessins animés qui en sont tirés.

63 Par exemple, les bipodes dont se sert l'armée de la République pour défendre la planète Kashyyyk sont moins élaborés dans leur partie supérieure que le modèle "ultérieur" plus volumineux que possède l'Empire dès l'épisode $V$. C'est en quelque sorte un état préalable de l'engin qui laisse supposer que, dans le monde du film, les ingénieurs impériaux ont élaboré plus tard un blindage permettant au pilote d'être entièrement dissimulé dans la "tête" de la machine.
Il découle de ce statut de prequel que l'évolution technologique dont ont bénéficié les films plus récents sert paradoxalement la représentation d'un univers censé être moins avancé sur le plan des technologies. Cette inversion est néanmoins motivée par les deux types d'univers dépeints, chacun se référant à un contexte spécifique: alors que la première trilogie renvoie explicitement à la Seconde Guerre mondiale (casques et tenues des officiers impériaux, tourelles de tir de type «bombardier» du vaisseau de Han Solo, etc.), la seconde décrit par contre, du moins jusqu'à la bataille de Géonosis, un univers d'heroic fantasy plus polissé dans lequel se développe la romance entre Anakin (Hayden Christensen) et Amidala (Natalie Portman). On peut donc motiver ces changements par la chronologie diégétique: les carlingues rutilantes des vaisseaux de la République (notamment celle du croiseur royal, entièrement argentée), les intérieurs cossus de Coruscant, les galbes prononcés des véhicules et architectures sous-marins de Gunga City et les cités lumineuses et italianisantes (ville de Theed) font place aux maquettes grisâtres des trois "premiers" épisodes, qui figurent des machines salies et supposément détériorées par des années de combats 61 . Si le "réalisme" de la représentation est peut-être inférieur dans la trilogie originale en termes d'invisibilité des effets spéciaux, il l'est beaucoup plus au niveau des caractéristiques visuelles des objets mécaniques, qui apparaissent véritablement comme des constructions (rivets et jointures apparents, omniprésence de ferrailles destinées à la déchèterie, etc.). De plus, les éléments machiniques y sont connotés sur le plan acoustique comme des produits de l'industrie lourde: la plupart des ambiances sonores - roulements sourds d'engrenages, frottements de tôles, cliquettement de mécanismes grippés, martèlements de pistons, sifflements de tuyères d'éjection de vapeurs - évoquent plus la Révolution industrielle de la fin du XIX ${ }^{\mathrm{e}}$ siècle que l'ère de l'informatique ${ }^{62}$. Les actions du robot R2-D2 renvoient certes à la dématérialisation et à la centralisation des données (sa fonction consiste invariablement à obtenir des informations cruciales en accédant à des "réseaux centraux"), mais ce personnage est simultanément présenté dans sa dimension matérielle, par exemple lorsqu'il se renverse en faisant un bruit sec de boîte de conserve.

En mêlant les styles graphiques des deux époques, La Revanche des Sith opère progressivement une jonction avec l'univers visuel de la trilogie fondatrice (notamment au niveau du design des véhicules) 63 qui culmine dans le plan où nous retrouvons un lieu et des protagonistes caractéristiques de l'épisode IV (Darth Vader et Tarkin). Cette image a probablement touché la plupart des spectateurs qui étaient familiers de la première trilogie, tant les époques (réelles) semblent se confondre, 
provoquant un fort effet de clôture64. C'est là l'une des particularités de la réception de l'univers filmique de Star Wars: l'écart inhabituel entre ces deux trilogies inscrites dans la continuité d'une même saga se lit inévitablement sur le plan du vécu des spectateurs. Le futur de ce passé diégétique des sequels qui nous apparaît dans le présent des sorties récentes est indéfectiblement lié au passé de certains spectateurs, ce qui réactive la nostalgie dont procédait la première trilogie par rapport à d'autres films plus anciens. Ce phénomène semble même intégré dans la conception des intrigues de la trilogie la plus récente: alors que l'épisode I nous montre Anakin à 8-10 ans et s'adresse sans équivoque à des kids, L'Attaque des clones correspond à l'adolescence et le dernier opus au passage à l'âge adulte (du moins tel que se le représente le cinéaste). Par ailleurs, l'un des ressorts narratifs principaux des intrigues de Star Wars repose sur l'idée de la filiation (entre maître et disciple, voire explicitement entre père et fils) ${ }^{65}$. Or la question du lien entre les générations se pose à l'extérieur de la diégèse, à travers l'écart temporel qui sépare la réalisation des différents films, puisque les enfants d'autrefois partagent aujourd'hui, grâce à l'univers Star Wars, un univers de référence, une sorte de "fonds commun" avec leurs propres enfants. A cet égard, le rapport du public aux films s'apparente à la «transmission orale» des mythes, fonction qui est d'ailleurs mise en abyme dans la première trilogie. En effet, bien que le drö̈de C-3PO déclare au tout début d'Un nouvel espoir à Luke, intéressé par les récits de batailles entre la Rébellion et l'Empire, qu'un robot comme lui ne sait pas raconter les histoires de façon captivante (il est en effet aussitôt interrompu), nous constatons au milieu du Retour $d u$ Jedi combien ce robot loquace a appris à devenir un conteur: entourés de petits Ewoks (doubles diégétiques des plus jeunes spectateurs), il "relate» les événements des épisodes précédents par une série de bruits qui, pour le public du film, sont immédiatement identifiables (comme les noms propres évoqués, seuls termes de cette langue imaginaire qui soient compréhensibles), tant chaque engin ou personnage est singularisé dans Star Wars sur le plan sonore. On peut dire que la projection d'un film de la série Star Wars sur grand écran endosse aujourd'hui le même rôle fédérateur et trans-générationnel que, par exemple, un concert des Rolling Stones.

Cette prise en compte du public (dont je ne nie pas les motivations mercantiles, mais qui relève également à mon sens de la responsabilité d'un "créateur de mythes») explique l'absence de scrupules que George Lucas manifeste envers les transformations apportées à l'ancienne trilogie. Il importe en effet que celle-ci corresponde aux normes du grand spectacle de la seconde moitié des années 1990 du point de vue du
64 Ainsi que le remarque Pierre Berthomieu: "L'architecture de cet épisode III qui soude définitivement les parties de la saga aboutit à des passages plus touchants encore pour leur puissance de nostalgie que pour leur (réelle) beauté propre" ("Les deux soleils de Tatooine ", in Positif, nº 533-534, juillet-août 2005, p. 126).

65 La question des rapports père-fils constitue, il est vrai, depuis les années 1980 une composante obsessionnelle du cinéma hollywoodien, mais la première trilogie Star Wars est particulièrement symptomatique de la prédominance de ce motif. 
66 La Trilogie Star Wars. Editions spéciales, dossier de presse, p. 10.

67 Laurent Jullier, L'Ecran post-moderne, op. cit., p. 38.

68 Umberto Eco, Lector in fabula. Le rôle du lecteur, Grasset, Paris, 1985 (1979), p. 33.

69 A l'exemple du livre de Bill Slavicsek paru avant la réalisation de la seconde trilogie (Star War Universe, Ballantine Books, New York, 1994) qui, l'année suivant sa première édition, portait déjà en couverture la mention "revised and expanded " qui s'applique aussi à l'univers dont il rend compte. Significativement, pour chaque entrée de ce dictionnaire, l'auteur précise si le mot se rapporte à une source originale de Lucasfilm (films, drames radiophoniques ou novellisations) ou à une source reconnue par cette instance (" an officially licensed source"). En France, les éditions Nathan ont édité à l'époque de la sortie des épisodes I, II et III des ouvrages de LucasBooks présentant grâce à de riches illustrations la quasi-intégralité des éléments appartenant au monde des films (costumes, armes, ustensiles, personnages, etc.), y compris en proposant des documents qui provoquent un "effet de réel" indéniable, par exemple des plans précis de vaisseaux spatiaux ou de complexes architecturaux. son (désormais en digital et THX) et des effets visuels (d'où l'utilisation de l'image numérique). Peu avant la sortie française des éditions spéciales qui eut lieu entre mars et avril 1997, le dossier de presse expliquait ainsi l'objectif de cette sortie:

"George Lucas était conscient que des millions d'adolescents, qui comptent certains des plus fervents admirateurs de la saga, n'avaient jamais eu la possibilité de découvrir leurs films préférés dans leur format original, au cinéma. Il leur manquait l'expérience inimitable du film vécu en commun avec plusieurs centaines d'autres personnes [...]. [Comme le dit Lucas:] ‘C'est le même genre d'expérience de groupe, de sentiment commun qui naît lorsqu'on assiste à un match de football ou à un concert de rock».»66

Les Star Wars constituent donc, dans l'esprit de Lucas, un lieu d'exaltation de la culture de masse. Le dernier comparant qu'il mentionne nous ramène, du point de vue cette fois de l'ensemble du public, à l'exemple des Rolling Stones. D'ailleurs, dans son ouvrage L'Ecran post-moderne, Jullier utilise l'expression "film-concert» pour qualifier les films qui fonctionnent exclusivement sur la délivrance de purs stimuli, et cite significativement le film Rolling Stones at the Max (1991)67.

\section{Star Wars comme "monde possible"}

Le statut de prequels des épisodes réalisés récemment pourrait mettre en péril la cohérence de l'univers diégétique de Star Wars posée dans la trilogie fondatrice. Tel n'est bien sûr pas le cas, Lucasfilm Ltd. exerçant un contrôle sévère sur l'ensemble des productions affiliées à Star Wars, fournissant une sorte de garantie d'authenticité à celles dont les récits se développent en conformité avec tout ce qui a été produit et agréé préalablement (il y a un "univers étendu» officiel). La nécessité évoquée par Umberto Eco pour le lecteur d'un roman de recourir à son «encyclopédie» (ensemble de connaissances sur la "réalité», construites par les discours ou acquises de façon empirique) $\mathbf{6 8}$ pour comprendre tout univers de fiction fonctionne dans Star Wars en autarcie par rapport au monde réel: il existe en effet un savoir «encyclopédique" relatif aux personnages, peuples, véhicules, planètes etc. de l'univers étendu, qui se concrétise dans de nombreux dictionnaires 69, sites ou jeux (l'existence d'un Trivial Pursuit Star Wars est tout à fait symptomatique de ce déplacement vers un monde fictionnel), et se manifeste à travers la nomination et la catégorisation des "états de chose» de ce monde (voir mon article sur les figurines qui clôt le présent dossier). On arguera qu'il existe d'autres phénomènes similaires, par exemple celui qu’a suscité Le Seigneur des anneaux de Tolkien (ouvrage dont l'influence est sensible 
dans le récit de Lucas). Néanmoins, dans Star Wars, les ramifications ont cela de particulier qu'elles ont été exclusivement générées par des productions cinématographiques (et leurs dérivés directs : pièces radiophoniques, adaptations romanesques ${ }^{70}$ ), de sorte que tout prolongement sera inévitablement ramené à ces dernières, et bien sûr évalué au niveau de sa concordance avec l'univers diégétique des films.

Or l'hexalogie est elle-même le résultat d'un ajout ultérieur de trois films, qui entraîne le respect d'une continuité avec la trilogie originale. On peut certes chercher l'infime détail qui, dans les films, nuirait à la perfection de la cohérence de ce monde, mais ce sont plutôt les raisons mêmes de la facilité avec laquelle cette dernière s'instaure qui me paraissent intéressantes. En effet, si l'univers de la première trilogie s'imbrique si bien dans celui de la seconde, c'est principalement à mon sens parce que l'on passe, comparativement, du vide au plein, et non l'inverse : le monde résultant des films réalisés entre 1977 et 1983 connaît effectivement un ameublement passablement inférieur à celui des productions ultérieures $\mathbf{7 1}$. Dans les deux premiers épisodes (IV et V) surtout, la quantité d'informants $\mathbf{7 2}$ livrés sur le monde est non seulement inférieure en termes de composants diégétiques montrés à l'image (les plans sont moins denses) - rappelons que Lucas avait même exploité à l'extrême une démarche soustractive dans $T H X 1138$, où les environnements se réduisaient à des surfaces blanches -, mais aussi au niveau de ce que l'on apprend via les dialogues. Ainsi dans Un nouvel espoir, seule la séquence de réunion de l'état-major de l'Empire permet d'entrevoir brièvement la face immergée de l'iceberg que constitue cette société fictionnelle (on apprend qu'un empereur - qui n'est pas encore l'Empereur (Palpatine), personnage-clé de la saga - a dissout le conseil du Sénat et élu des gouverneurs régionaux). La hiérarchie complète de cette société demeure hors-film (ce qui accentue le caractère manichéen de la représentation), comme nombre d'éléments dans l'image. Un exemple: lorsque, dans l'épisode IV, Luke et Han neutralisent deux gardes impériaux pour leur subtiliser leurs uniformes et leurs casques, la séquence est filmée comme dans une comédie burlesque, puisque l'on entend hors-champ les coups de "blasters" avant de voir réapparaître le garde, auquel on imagine que l'un des héros s'est substitué (sans mesurer l'amplitude de l'ellipse, qui permet à cet instant une accélération du tempo narratif). Si le film ne tenait pas tant à évacuer tous les moments creux et si nous avions vu, côte à côte, les deux gardes démasqués dans la soute du vaisseau, il n'aurait plus été possible de concevoir ultérieurement ces soldats comme des clones qui possèdent tous le même visage, celui du père du chasseur de primes Boba Fett (qui lui aussi n'est jamais montré sans son
70 En novembre 1976 sort un roman intitulé Star Wars, signé "George Lucas". Six mois plus tard, alors que le film en est encore au stade de la post-production, pas moins d'un demi million d'exemplaires de cet ouvrage sont vendus. Cette donnée permet de relativiser l'idée souvent reprise selon laquelle le succès du film de 1977 fut une surprise complète pour Lucas et la Fox (voir L'Empire des rêves. L'histoire de la trilogie Star Wars).

71 Sans que cet article soit le lieu d'une discussion de la théorie des mondes possibles, j'emprunte à ce cadre de réflexion les principaux concepts mentionnés ici. Pour plus de précisions, je renvoie aux travaux d'Umberto Eco et de Thomas Pavel, ou à mon ouvrage La Fiction au cinéma, op. cit.

72 Afin de concevoir la notion d'، univers filmique" dans une optique communicationnelle, j'ai proposé dans La Fiction au cinéma (op. cit., pp. 125-130) d'identifier dans les films des éléments qui livrent plus ou moins ostensiblement des indications sur cet univers au spectateur, à partir desquelles ce dernier peut effectuer des inférences de diverses natures. 
casque dans la première trilogie). L'économie narrative plutôt «suppressive» des films plus anciens de la saga - l'une des rares planètes à être nommée dans l'épisode IV, Alderaan, est significativement rayée de la carte par le canon de l'Etoile de la Mort - offre rétrospectivement des lacunes favorables à l'élaboration du scénario des prequels. En outre, la «sous-humanité» de plusieurs personnages principaux qui traversent les deux trilogies permet de justifier certains éléments qui pourraient faire obstacle à la continuité : ainsi la mémoire des droïdes est-elle effacée à la fin de La Revanche des Sith, et l'on ne s'étonne guère que le gorille Chewbacca, qui a combattu aux côtés de Yoda contre la Fédération du Commerce et fut aux premières loges de l'éradication des Jedi, ne fasse jamais mention de ce passé lorsque, devenu copilote du «Faucon Millénium ", il fait la rencontre d'Obi-Wan Kenobi, ex-général (à nouveau, la discussion entre eux demeure hors-champ à la suite d'un contrechamp sur Luke qui détourne le regard): ne communiquant que par grognements, Chewbacca ne véhicule que peu d'informants narratifs.

Les concepteurs de la saga Star Wars ont exploité le potentiel offert par un espace (galactique) totalement inconnu du spectateur, c'est-à-dire susceptible de révéler à tout moment une nouvelle galaxie, de nouveaux peuples, etc. Chaque épisode s'ouvre d'ailleurs sur une vue de l'espace cosmique, étendue infinie ouverte à tous les possibles, à toutes les "projections» de la part du public et de ses créateurs. Cette actualisation s'opère alors dans la périphérie des films, voire au sein même du public, ainsi que le note Laurent Jullier:

«Star Wars est une fiction en expansion constante, dont les deux trilogies cinéma ne constituent que la petite partie centrale. Des centaines de milliers de fans à travers le monde et quelques milliers d'auteurs professionnels de jeu, de BD et de romans travaillent quotidiennement à fabriquer ses ramifications, ses mondes possibles, ses déclinaisons. » $\mathbf{7 3}$

Dès lors, l'œuvre filmique n'est plus qu'un pré-texte, l'amorce d'un «texte»- au sens originaire de «tissu, réseau (de sens)»- qui s'échafaude ailleurs, en obéissant à des procédures de consommation de l'image différentes de celles qui définissent l'institution cinématographique.

\section{L'extension perpétuelle: l'exemple des comics}

Les productions extrafilmiques qui donnent naissance à l'univers étendu complexifient l'extrême linéarité de la temporalité diégétique des films (que seuls viennent rompre les flash-backs sonores et les cauchemars prémonitoires d'Anakin dans La Revanche des Sith), ainsi que les rapports entre les différentes étapes de l'expansion du monde et le vécu 
des spectateurs (qui sont également, selon les cas, lecteurs, auditeurs et joueurs). En situant quelques bandes dessinées sur l'axe d'une double chronologie (diégétique et réelle), j'essaie de rendre compte dans l'annexe (voir pages 38 à 41 du présent dossier) comment interagissent ou s'imbriquent les nombreux ancrages temporels. Si l'on s'intéresse aux dates de parution des comics - support intéressant dans la mesure où il est à la fois aussi narratif qu'un roman et presque aussi figuratif qu'un film ou qu'un jeu vidéo -, on constate tout d'abord une période d'exploitation immédiate du succès d'Un nouvel espoir et de ses deux sequels: Marvel Comics publie entre 1977 et 1986 pas moins de 107 numéros liés à Star Wars. Si les sept premiers racontent de façon synthétique l'histoire de l'épisode IV sur le mode de l'"adaptation", les suivants bifurquent sur des aventures postérieures ou parallèles au récit fondateur (la mention de la couverture passe de "The Greatest Space-fantasy Film of all!» à "At last! Beyond the movie! Beyond the galaxy!"). "Au-delà du film": non seulement la $\mathrm{BD}$ permet de conserver quelque chose du film après sa disparition des salles (à une époque antérieure à la généralisation des moyens du visionnage à domicile), mais elle s'autonomise sur le plan narratif. Il s'agit là d'un tournant non seulement par rapport à la ligne d'intrigue conçue par George Lucas, mais aussi au niveau des liens instaurés entre ce blockbuster et les productions qui en furent dérivées. Dans la seconde moitié des années 1990, on observe un véritable essor des comics Star Wars, probablement consécutif au succès des trois romans de La Trilogie de Thrawn écrit par Timothy Zahn, auteur de sciencefiction confirmé. En 1992-1993, il révèle l'intérêt (et la rentabilité !) de l'expansion de l'univers Star Wars en racontant les aventures des héros de la trilogie originale situées dans la chronologie diégétique après $L e$ Retour du Jedi, lorsqu'un amiral tente de reconstituer une armée impériale disséminée. L'adaptation bédéique de ces romans paraît dès 1996, simultanément à la publication de l'autre roman qui déclencha une prolifération des supports médiatiques autour d'un même récit, Shadows of the Empire de Steve Perry 74. En effet, Les Ombres de l'Empire s'est vu décliné sous toutes ses formes: en cette même année 1996 apparaissent sur le marché les deux tomes de l'adaptation de l'ouvrage en comic book, une version du roman pour la jeunesse écrite par Christopher Golden, un jeu vidéo pour la console Nintendo Ultra-64, des figurines chez Kenner dérivées de la $\mathrm{BD}$ et, même, une musique éditée sur $\mathrm{CD}$ chez Varèse Sarabande Records, composée par Joel McNeely sur le modèle "post-wagnérien» de Williams, avec par exemple un thème lié au méchant Xizor. Composer une musique pour un livre en sélectionnant des moments narratifs centraux (des «séquences» musicales), fabriquer
74 L'importance de ces récits situés peu après Le Retour du Jedi restreindrait d'ailleurs passablement le travail scénaristique de Lucas sur une troisième trilogie inscrite dans la continuité des deux premières. On comprend dès lors qu'il ait choisi de s'en tenir aux sequels, sans quoi ce serait le "paratextuel " qui dicterait ses lois au texte filmique (à moins d'être oblitéré). 
75 Notons que toutes les productions qui s'inscrivent dans la masse parafilmique de Star Wars ne présentent pas, ainsi que l'a montré Will Brooker dans son analyse du discours des fans, le même degré de canonicité. La détermination de ce degré obéit au principe de l'auteurisme: plus les récits sont proches de ceux que l'on attribue à Lucas, plus la probabilité de leur actualisation "effective" (dans la fiction) est considérée comme élevée (Will Brooker, "Internet fandom...", op. cit., p. 53 et p. 67). Brooker cite une correspondance avec un fan où ce dernier explicite cette hiérarchie: "Zahn s'est produit, au moins sa trilogie originale. Certains événements contés par Anderson ont pu avoir lieu. [...] Mclntyre ne s'est pas produit!" (id., p. 68). Ces jugements sur la collaboration de différents auteurs à l'univers étendu sont révélateurs du primat inconditionnel accordé aux films sur le paratexte, de même que la quasiassimilation de l'intrigue canonique à une certaine "réalité" qui montre combien les films sont lus en tant qu'instruments d'élaboration d'un "monde".

76 Ici comme dans l'annexe, il n'est jamais question du style graphique des comics mentionnés. Cette limitation ne doit préjuger en rien du manque d'intérêt de celui-ci, mais la multitude d'artistes qui sont intervenus sur ces séries m'a contraint à m'en tenir au contenu narratif, c'est-à-dire à une dimension sémantique directement liée à la question des "mondes" sur laquelle je mets l'accent. des jouets liés à un univers filmique qui est demeuré virtuel: dans la seconde moitié des années 1990, l'univers Star Wars a connu un mouvement d'expansion qui n'a plus tari depuis. Il n'est pas étonnant de constater que c'est précisément à cette époque que Lucasfilm Ltd. s'investit dans la restauration de la trilogie fondatrice, prévoit une ressortie en salles et conçoit la réalisation des prequels. Le développement des ramifications extrafilmiques de Star Wars a probablement joué un rôle dans l'existence même des sequels qui, au même titre que ces productions parallèles, comblent les trous suggérés par les références au passé dans la trilogie fondatrice.

Il n'est bien sûr pas envisageable d'aborder dans le cadre de cette introduction cette production de comics qui s'avère, du moins dans le domaine anglophone, véritablement pléthorique. On retiendra cependant en conclusion un triptyque hautement révélateur de la façon dont les récits extracinématographiques de Star Wars déploient l'univers premier de la saga filmique: il s'agit de trois comic books portant le même titre que chacun des épisodes de la trilogie fondatrice, mais racontant un récit alternatif par rapport à celui qui nous a été montré sur les écrans. Cette démarche évoque a priori plus le schéma arborescent sur lequel se base le diptyque adapté d'Ayckbourn Smoking/No Smoking (Alain Resnais, 1993) qu'elle n'illustre le souci de cohérence absolue dont témoignent généralement les garants de l'univers Star Wars. Toutefois, elle n'échappe pas au contrôle de Lucasfilm, qui crée en 2001 pour sa filiale LucasBooks et pour Dark Horse Comics le label «Infinities». Cette «marque déposée» désigne toutes les productions présentant certains contenus narratifs susceptibles de s'avérer contingents (au sens où ils peuvent ne pas être), c'est-à-dire que le lecteur ne doit pas intégrer dans l'univers étendu préalablement fixé75. Ouvrant des possibles au sein même d'un "monde possible", ce type d'ouvrages entretient des liens d'autant plus étroits avec les films lorsqu'il s'agit de récits "sérieux", et non de variations parodiques qui, elles, sont ouvertement distinctes de la fiction des films. Ainsi, Star Wars Infinities. A New Hope (non traduit en français à ce jour) scénarisé par Chris Warner (2001) 76 inaugure une trilogie parallèle. Après avoir synthétisé sur trois planches le film Un nouvel espoir en choisissant une série d'images-clés de cet épisode accompagnées d'un récitatif (mais sans phylactères, comme une voixover qui commenterait une bande-annonce), le récit verbal se termine par l'affirmation suivante: "Altérez un seul événement, et un nouveau futur naîtra». Cette version "Infinities» ne transforme donc pas de fond en comble l'histoire originelle, mais opte pour des bifurcations en des points bien précis de l'évolution causale du récit écrit par Lucas. La 
déviation première dont découlent toutes les autres est la suivante: Luke Skywalker n'étant pas parvenu à faire pénétrer le missile dans le conduit de l'Etoile de la Mort - échec dévirilisant s'il en est ! -, la base spatiale est en mesure d'anéantir la plus grande partie de l'Alliance Rebelle. Plus tard, on assistera à un défilé de l'armée impériale durant lequel Leia Organa, debout sur une tribune, tend le bras en signe de salut (nazi) au passage des troupes, puis tient un discours élogieux sur les effets bénéfiques de la victoire de l'Empire. Les dangers d'une dérive fasciste au sein même de l'équipe des héros, latents dans les films, sont donc représentés de façon explicite dans ce récit, comme si les éléments sousjacents relevés par Robin Wood avaient été explicitement intégrés au contenu narratif. Même les diatribes se voient phagocytées par l'univers Star Wars à travers la formule "Infinities» (selon une logique de réappropriation maligne des discours qui est propre à Star Wars en général) : si Wood pointe le fait que les personnages féminins n'héritent que de rôles mineurs, soulignant par exemple à propos de la princesse Leia "qu'il n'est jamais fait allusion au fait qu'elle puisse hériter de la Force» $\mathbf{7 7}$, la bande dessinée Star Wars Infinities. The Empire Strikes Back (2003) fait mourir Luke dans les neiges de Hoth, ce qui conduit Leia à suivre l'entraînement de Maître Yoda pour devenir une Jedi. Il est vrai que les comics de Star Wars font par rapport aux films la part belle aux personnages féminins - on constate là l'influence patente des mangas et films d'arts martiaux asiatiques -, à l'exemple de la série Mara Jade. Les réalisations bédéiques récentes introduisent plusieurs personnages de guerrières ou de Jedi femmes qui demeurent à l'arrière-plan (aux sens tant littéral que figuré) dans la nouvelle trilogie filmique où, cantonnées à faire de la figuration, elles n'accèdent significativement pas à la parole (lieu d'expression d'un pouvoir réservé aux hommes). L'unique protagoniste qui, créé dans l'univers étendu, a ensuite rejoint les films (au-delà de la seule mention verbale de son nom) est un personnage féminin, Aayla Secura. Or elle n'occupe dans La Revanche des Sith un rôle central que dans un fragment de cinq plans continus au cours duquel elle demeure muette, et qui représente précisément son assassinat par les clones, c'est-à-dire son éviction du film (une façon pour Lucas de signifier qu'il campe sur ses positions d'antan) $\mathbf{7 8}$.

On l'aura compris, les productions parallèles aux films permettent d'éviter ou de prévenir rétrospectivement presque toutes les critiques d'alors, si ce n'est celle de ressortir à ce que Wood appelle le "paradigme de la répétition ", qui consiste à proposer «la même formule, mais avec des variations» $\mathbf{7 9}$. Les "Infinities» portent en effet à son plus haut point cette logique de répétition/variation qui présida à la genèse d'un
77 Robin Wood, op. cit., p. 173.

78 On arguera certes que Lucas a supprimé au montage de La Revanche des Sith une partie de la longue séquence d'ouverture qui comprenait le personnage féminin de la Jedi Shaak Ti, passage désormais intégré aux suppléments de l'édition DVD. II faut noter toutefois que la fonction de ce personnage se limite à celle d'un appât destiné à faire entrer en scène les deux héros masculins: une fois que ceux-ci se sont approchés, le Général Grievous abat Shaak Ti, qui n'a prononcé qu'une seule réplique empreinte de déférence et d'un sentiment d'infériorité envers Obi-Wan ("Je suis désolé, Maître Kenobi, j'ai échoué").

79 Robin Wood, op. cit., p. 163. 
épisode par rapport au précédent (ou de la nouvelle trilogie par rapport à l'ancienne). Le label choisi par LucasBooks pour qualifier ce type de productions l'indique clairement: tout est réitérable à l'infini! L'histoire des films continue ainsi d'exister à travers des avatars qui réaffirment ad libitum (ou ad nauseam pour certains) son existence. L'actualisation des

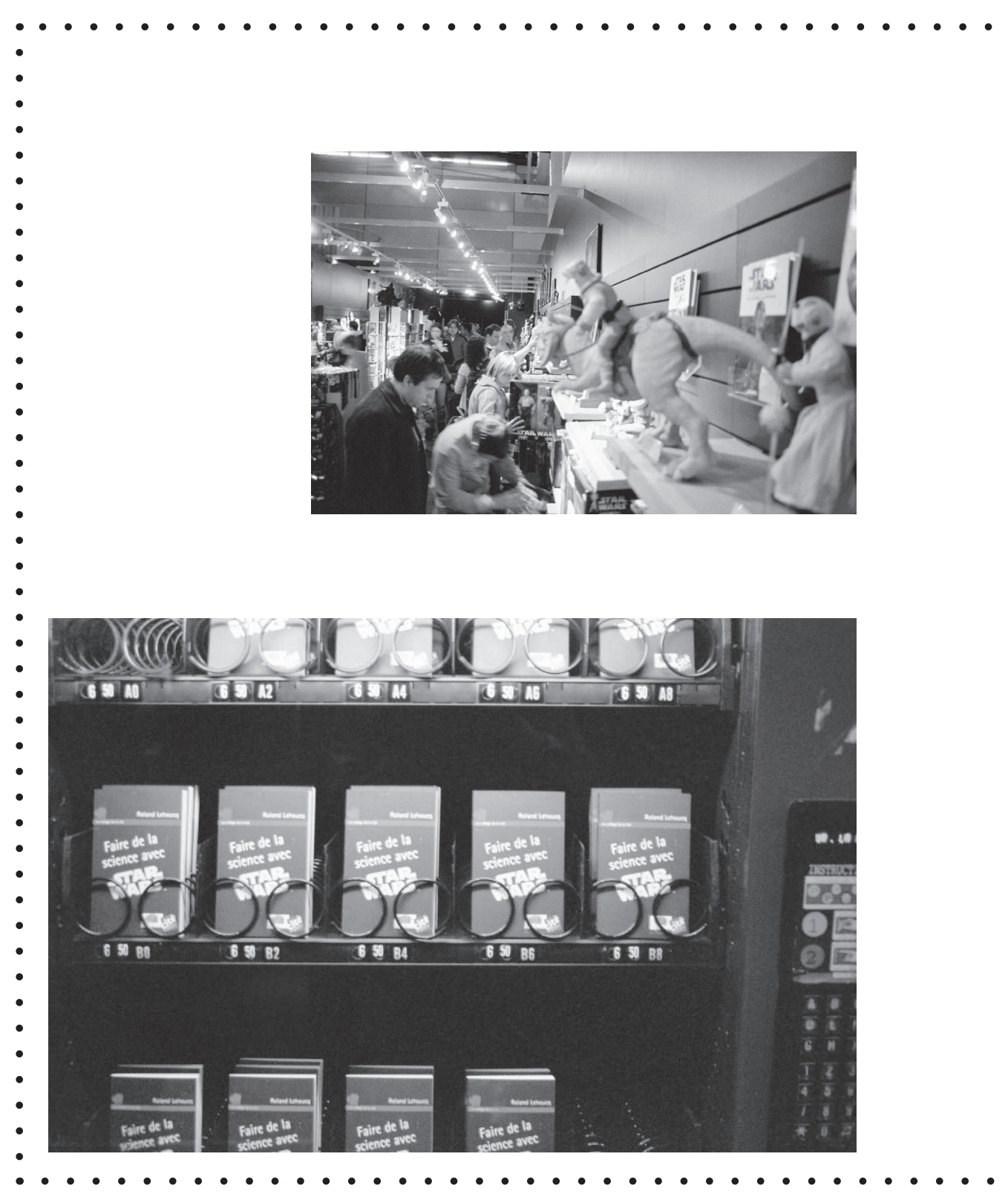


possibles permet à l'assise commune des films d'être toujours actuelle. Trente ans après la sortie d'Un nouvel espoir, on peut dire qu'à l'extérieur du champ cinématographique, l'univers Star Wars n'a pas fini de faire parler de lui...

\section{Star Wars comme phénomène: l'exposition parisienne}

L'exposition qui s'est déroulée du 18 octobre 2005 au 27 août 2006 à la Cité des sciences et de l'industrie de Paris a révélé combien toute manifestation relative à Star Wars a tôt fait de se voir aspirée dans le gouffre de la promotion. Exhibition d'objets utilisés pour la conception ou la réalisation des films (costumes, maquettes, illustrations), cette exposition n'a pas vraiment construit de discours sur le phénomène Star Wars, mais nous a fait pénétrer dans ses différents "mondes", au gré d'espaces dans lesquels retentissaient les bruits et la musique des films. On constate donc qu'une telle manifestation n'échappe pas à "l'immersion" du spectateur exploitée dans toutes les productions périphériques aux films. Cette caractéristique touche également la dernière salle de l'exposition, qui n'est autre qu'un magasin où l'on vend les produits dérivés. La démarche qui consiste à annexer ainsi le secteur des ventes (au grand dam des parents lorsqu'ils constatent les prix prohibitifs) à un lieu supposément culturel et éducatif afin d'exploiter le conditionnement opéré sur les plus jeunes par l'exposition est certes révélatrice du mercantilisme foncier de "l'Empire Lucas", mais entre également en résonance avec le "contenu" des salles précédentes, d'un intérêt tout relatif: la véritable exposition ne serait-elle pas autre chose qu'un magasin de jouets? Ou, pour renverser l'équation en se déprenant du mépris communément manifesté dans le champ des études sur le cinéma envers le merchandising: les produits dérivés ne constituent-ils pas le véritable sujet de l'exposition? Pour la première trilogie du moins (réalisée à l'ère des maquettes et des effets sonores, lumineux et pyrotechniques), la différence entre les éléments "profilmiques" conservés et les engins miniatures vendus dans le commerce est minime. Car Star Wars, c'est avant tout cela: un ensemble d'icônes de la culture de masse, reconnaissables par la plus grande majorité des spectateurs et multipliables à l'envi sous quantité de formes. II est toutefois intéressant que l'exposition parisienne se soit bornée à réduire Star Wars à cette dimension fétichiste. Dans les Star Wars, le "filmique" (mise en scène, montage, photographie) n'y importe pas autant que le design et le travail graphique des différents collaborateurs qui naissent sur d'autres supports (la planche à dessin, la maquette), se concrétisent à l'écran avant d'être à nouveau diffusés sous une forme proche de ce qu'ils furent originellement. L'exposition montre que Star Wars se laisse difficilement détourner de la vacuité ludique de l'autodésignation (on n'apprend bien moins que l'on reconnaît). Il y a certes l'opuscule de vulgarisation scientifique commandé à Laurent Lehoucq, Faire de la science avec Star Wars: vendu sur un mode "mécanique" totalement adapté à son objet (il était vendu dans des automates situés dans les couloirs de la Cité des sciences), cet ouvrage bute toutefois sur le caractère passablement fantaisiste de l'univers Star Wars, décomplexé face à toute vraisemblance scientifique (en cela, la première trilogie se distinguait radicalement de 2001, l'Odyssée de l'espace réalisé dans la décennie précédente): partir de la Force qui anime les Jedi pour traiter de champs électrique et gravitationnel, ou estimer les dimensions de la planète Endor du Retour du Jedi (en faisant remarquer que les soldats déployés par l'Alliance n'y progressent pas par bonds comme des astronautes sur la lune) ne fait que relever par contraste la désinvolture avec laquelle ce space opera se plie aux lois de notre monde physique. L'univers de Star Wars se laisse difficilement exploiter à des fins pédagogiques: le "savoir" que l'on peut transmettre à travers les représentations qui en découlent demeure foncièrement défini par le fort degré de fictionalité qui caractérise ce monde.

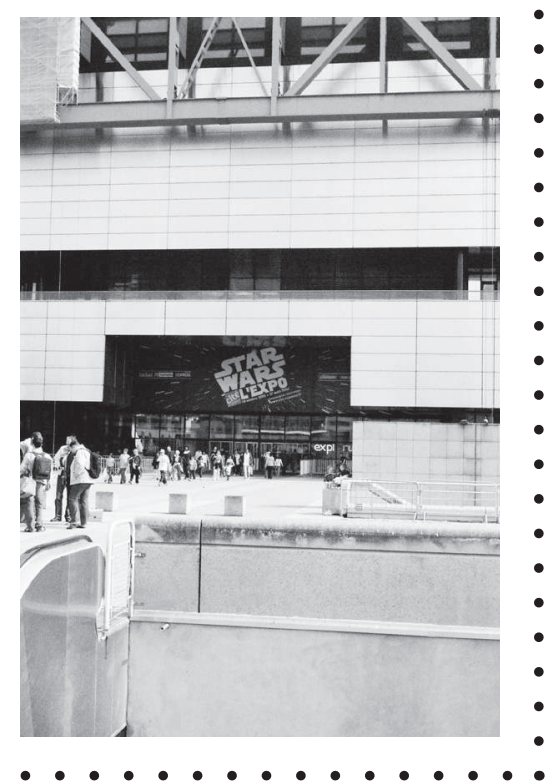


Depuis la réalisation des prequels à la fin des années 1990, les tentatives d'établir une chronologie des multiples intrigues suscitées à partir de l'univers Star Wars furent nombreuses, tant sur des sites Internet que dans des articles de presse. Comme il existe de nos jours près d'un millier d'ouvrages offrant des récits affiliés à Star Wars (bandes dessinées et romans), il est bien sûr difficile d'en rendre compte de manière satisfaisante sans user de la présentation par liens et entrées que permet Internet. En me cantonnant à un choix de quelques comics qui me paraissent importants du point de vue de l'enrichissement de l'univers étendu - j'ai notamment écarté avec partialité ceux qui développent les aventures d'un personnage singulier déjà présent dans les films (Chewbacca, Boba Fett, Han Solo, etc.), et même l'intégralité de ceux qui ont trait, comme un film TV et une série d'animation (1985-1987), à la peuplade d'oursons appelés Ewoks -, j'aimerais toutefois proposer à mon tour une chronologie synthétique et partielle en mettant l'accent sur une dimension généralement évacuée par les commentateurs, pour la plupart entièrement plongés dans l'univers Star Wars comme s'il ne résultait pas d'une série de productions elles-mêmes inscrites dans une chronologie. Il s'agit des dates de parution des divers opus. En effet, dans ce monde en perpétuelle expansion, la lecture des "spectateurs" varie en fonction de ce qui a été publié précédemment, de même que la lecture des événements diégétiques faite par certains auteurs dépend des données qui ont été fixées par les films. En mettant en regard l'époque où se déroulent les actions et la date de réalisation du «texte» qui les relate, on peut saisir la complexité de la participation du narrataire à l'élaboration mentale de l'univers Star Wars. L'importance de cette chronologie factuelle s'avère même capitale pour la création d'événements fictionnels parallèles à la continuité de référence posée par les six films : par exemple, si le Darth Vader de Star Wars Infinities. The Empire Strikes Back peut accéder à la mémoire du droïde C-3PO et apprend ainsi que sa cible se trouve sur la planète Dagobah (où il apparaîtra ensuite physiquement, et non seulement dans un cauchemar comme dans le film), c'est, ainsi qu'il l'affirme, parce qu'il a lui-même conçu ce robot. Or, tandis que ces événements sont situés à l'époque de l'Episode $V$ (1980), l'information selon laquelle le jeune Anakin Skywalker a construit de ses mains C-3PO n'apparaît que dans l'Episode I (1999) : la bande dessinée ayant été réalisée en 2003, son auteur peut ainsi intégrer un élément supplémentaire du passé du personnage d'Anakin/Vader. Cet entremêlement des deux types de chronologie est constant et fait la richesse de certains de ces récits. En outre, le point d'insertion d'un récit donné dans la continuité de Star Wars n'est pas forcément unique, dans la mesure où toutes les histoires ne sont pas nécessairement aussi linéaires que les films. Ainsi dans Crimson Empire, comic book scénarisé par Mike Richardson et Randey Stradley (Dark Horse, 1998) dans lequel nous adoptons le point de vue d'un ancien membre de la garde royale de l'Empereur plusieurs années après la chute de ce dernier, un flash-back nous fait remonter à l'époque de la formation du soldat (il s'agit d'une tournée d'inspection de Palpatine et Vader), convoquant ainsi une période ancrée dans la temporalité du film Le Retour du Jedi.

Il faut noter que les productions appartenant à l'univers étendu de Star Wars situent très précisément les récits sur l'axe de la chronologie diégétique jalonnée par les six films. Le point de repère principal est invariablement la "Bataille de Yavin", c'est-à-dire la destruction de la première Etoile Noire à la fin d'Un 
nouvel espoir, moment qui constitue, à l'instar de la nativité dans le calendrier grégorien, l'année zéro - on dira par exemple que La Menace fantôme se passe en 32 BBY ("Before the Battle of Yavin»). Cette rationalisation de la temporalité de Star Wars a deux conséquences: d'une part, elle tend à créer une confusion entre le temps diégétique et celui de la production, puisque Yavin renvoie au film de 1977, origine effective de la saga; d'autre part, elle s'oppose à l'indécision de l'indication "Il y a bien longtemps..." sur laquelle s'ouvre chaque film, comme si la fable intemporelle devait se plier aux desiderata d'un historien méticuleux. Les dates-clés, systématiquement relatives à des combats comme dans une histoire militaire, donnent par contre une assise commune qui facilite la transmission des divers prolongements narratifs; elles sont de toute manière de pure convention, s'expliquant dans certains cas par une approximation de l'âge des protagonistes en fonction de celui des acteurs dans les films (ainsi décide-t-on "officiellement» que dix ans séparent l'épisode I du II). L'anthologie en dix albums des Clone Wars, éditée en français chez Delcourt, s'inscrit ainsi dans une portion de temps nettement déterminée: au début des volumes 1 à 6 , on nous précise à la semaine ou au mois près quand se passent les événements contés par rapport à la bataille de Géonosis (visualisée dans L'Attaque des Clones); du numéro 7 au 9, on nous informe que les actions se déroulent environ cinq ou six mois avant La Revanche des Sith. Le point de repère change, annonçant la convergence $\mathrm{du}$ tome 10, dont les faits sont simultanés aux aventures du dernier film tourné (il s'agit de la débâcle des Jedis, supprimés par leurs propres troupes conséquemment à «l'ordre 66» de Palpatine) sans pour autant que l'on assiste à un récit «répétitif» au sens de Genette: à l'exception d'une planche consacrée à la décision de Yoda de fuir avec l'aide de deux indigènes, tout ce qui est montré et dit se situe entre les "mailles» du texte filmique, le complète sans le contredire.

L'établissement d'une chronologie factuelle des productions Star Wars doit par ailleurs tenir compte de l'existence de différents états des textes ou de variations dans les modes de parution. Ainsi chaque film de la trilogie originale sort-il en trois versions à trois époques différentes, et certains récits dessinés paraissent d'abord dans des journaux sous forme de strips avant d'être repris en comic books. Concernant ces derniers, on distinguera également les versions anglaises publiées chez Dark Horse de leurs traductions françaises, qui paraissent parfois beaucoup plus tardivement et ne recueillent pas l'intégralité des récits parus aux Etats-Unis. 


\section{TITRES DANS L'ORDRE DE LA TEMPORALITÉ DIÉGÉTIQUE}

(Entre parenthèses: nom du scénariste)

\section{DATE(S) DE SORTIE}

(Editeurs de comics: M.= Marvel; $\mathrm{DH}=$ Dark Horse;

$\mathrm{DHF}=$ Dark Horse France $;$ Del. $=$ Delcourt $)$

$1997(\mathrm{DH}) ; 1998(\mathrm{DHF})$

1998 (DH; DHF)

$1994(\mathrm{DH})$

2001-2002 (DH)

\section{$\mathbf{3 0 0 0}$ ans plus tard}

Jedi vs Sith (Darko Macan)

$\mathbf{9 6 8}$ ans plus tard

Episode I - La Menace fantôme (George Lucas)

Adaptations en comics et manga

Durant les années suivantes

Nombreux Tales

10 ans après l'Episode I

Episode II - L'Attaque des clones (George Lucas, Jonathan Hales)

2002

Adaptations en comics

De quelques semaines à 3 ans après

Série Republic

En France: séries Clone Wars et Jedi

Star Wars : Clone Wars (série TV d'animation)

Graphisme de la série TV repris en comics :

Clone Wars Adventures

Episode III - La Revanche des Sith (George Lucas)

Adaptations en comics

Environ 15 ans plus tard

Série TV d'animation Droids

Environ 20 ans plus tard

Droids: série de comics consacrée à R2-D2 et C-3PO

1985-1986

2003-2006 (DH)

2004-2006 (Del.)

2003-2005

2005

1986 (M.) 


\section{Environ $\mathbf{3 0}$ ans plus tard}

Série de comics: Empire (n $\left.{ }^{\circ} 1-11\right)$ et certains Tales

2002-2003

Episode IV - Un Nouvel Espoir (George Lucas)

Adaptations en comics et manga

En même temps que l'Episode IV

Star Wars: Empire

Peu après

Vader's Quest (Darko Macan)

Classic Star Wars (Archie Goodwin)

Episode V-L'Empire contre-attaque (George Lucas, Leigh Brackett)

Adaptations en comics et manga

\section{Durant les mois suivants}

Shadows of the Empire (John Wagner, d'après Steve Perry)

1 an après l'Episode $\mathbf{V}$

Episode VI-Le Retour du Jedi (George Lucas, Lawrence Kasdan)

Adaptations en comics et manga

En même temps que l'Episode VI (puis juste après)

Mara Jade (Michael Stackpole, d'après Timothy Zahn)

Environ 1 an plus tard

Série $X$-Wing Rogue Squadron

$\mathbf{5}$ ans plus tard

Le Cycle de Thrawn (trilogie) (Mike Baron, d'après

Timothy Zahn) (L'Héritier de l'Empire; La Bataille des Jedi;

L'Ultime Commandement)

6 ans plus tard

Dark Empire (trilogie) (Tom Veitch)

1991 (DH) ; 2006 (Del.)

1977 ; 1997 (Special Ed.) ; 2004 (DVD)

1977 (M.); 1994 (DH) ; 1997 (DH);

1998 (Del. ; DH manga)

$2002(\mathrm{DH})$

1999 (DH); 2004 (Del.)

1981-1984 (M.) ; 1992-1996 (DH)

1980 ; 1997 (Special Ed.) ; 2004 (DVD)

1980-1981 (M.); 1994 (DH); 1997 (DH);

1999 (Del. ; DH manga)

1996 (DH; DHF); 2006 (Del.)

1983 ; 1997 (Special Ed.) ; 2004 (DVD)

1980-1981 (M.) ; 1994 (DH) ; 1997 (DH);

1999 (Del. ; DH manga)

1998-1999 (DH); 2006 (Del.)

1996 (DH); 2006 (Del.)

1997-1998 (DH; DHF); 2005 (Del.) 